TRANSACTIONS OF THE

AMERICAN MATHEMATICAL SOCIETY

Volume 351, Number 12, Pages 4905-4945

S 0002-9947(99)02418-6

Article electronically published on July 20, 1999

\title{
COMPLEX SYMPLECTIC GEOMETRY WITH APPLICATIONS TO ORDINARY DIFFERENTIAL OPERATORS
}

\author{
W. N. EVERITT AND L. MARKUS
}

Dedicated to Professor Hugh L. Turrittin on the occasion of his ninetieth birthday

\begin{abstract}
Complex symplectic spaces, and their Lagrangian subspaces, are defined in accord with motivations from Lagrangian classical dynamics and from linear ordinary differential operators; and then their basic algebraic properties are established. After these purely algebraic developments, an Appendix presents a related new result on the theory of self-adjoint operators in Hilbert spaces, and this provides an important application of the principal theorems.
\end{abstract}

\section{Fundamental Definitions FOR COMPLEX SyMPLECTiC SPACES, AND THREE MOTIVATING ILLUSTRATIONS}

Complex symplectic spaces, as defined below, are non-trivial generalizations of the real symplectic spaces of Lagrangian classical dynamics [AM], [MA]. Further, these complex spaces provide important algebraic structures clarifying the theory of boundary value problems of linear ordinary differential equations, and the theory of the associated self-adjoint linear operators on Hilbert spaces [AG], [DS], [NA].

These fundamental concepts are introduced in connection with three examples or motivating discussions in this first introductory section, with further technical details and applications presented in the Appendix at the end of this paper. The new algebraic results are given in the second and main section of this paper, which developes the principal theorems of the algebra of finite dimensional complex symplectic spaces and their Lagrangian subspaces. A preliminary treatment of these subjects, with full attention to the theory of self-adjoint operators, can be found in the earlier monograph of these authors [EM].

Definition 1. A complex symplectic space $S$ is a complex linear space, with a prescribed symplectic form [:], namely a sesquilinear form

$$
\text { (i) } u, v \rightarrow[u: v], \quad S \times S \rightarrow \mathbf{C} \text {, so }\left[c_{1} u+c_{2} v: w\right]=c_{1}[u: w]+c_{2}[v: w] \text {, }
$$

which is skew-Hermitian,

$$
[u: v]=-\overline{[v: u]}, \text { so }\left[u: c_{1} v+c_{2} w\right]=\bar{c}_{1}[u: v]+\bar{c}_{2}[u: w]
$$

Received by the editors August 19, 1997.

1991 Mathematics Subject Classification. Primary 34B05, 34L05; Secondary 47B25, 58F05.

Key words and phrases. Ordinary linear differential operators, deficiency indices, symmetric boundary conditions, symplectic geometry. 
(in terms of the conjugation involution $c \rightarrow \bar{c}$ in the complex number space $\mathbf{C}$ ), and which is also non-degenerate,

$$
\text { (iii) } \quad[u: S]=0 \text { implies } u=0 \text {, }
$$

for all vectors $u, v, w \in S$ and complex scalars $c_{1}, c_{2} \in \mathbf{C}$.

Because of the analogy of the complex symplectic space $S$ to a Hermitian inner product space, we often employ the terminology "symplectic product" for $[u: v]$ and "symplectic orthogonality" for $[u: v]=0$. Hence condition (iii) above means that only the zero vector of $S$ is symplectically orthogonal to every vector of $S$.

As is customary, we declare that complex symplectic spaces $S_{1}$ with form [:] and $S_{2}$ with form [:] $]_{2}$ are isomorphic in case there exists a linear bijective map

$$
F: S_{1} \rightarrow S_{2} \text { with }[u: v]_{1}=[F u: F v]_{2},
$$

for all vectors $u, v \in S_{1}$.

Linear subspaces of a complex symplectic space $S$ need not be complex symplectic subspaces, since the induced symplectic form can be degenerate on them.

Definition 2. A linear submanifold $L$ in the complex symplectic space $S$ is called Lagrangian in case $[L: L]=0$, that is,

$$
[u: v]=0 \text { for all vectors } u, v \in L \text {. }
$$

Further, a Lagrangian manifold $L \subset S$ is complete in case

$$
u \in S \text { and }[u: L]=0 \text { imply } u \in L .
$$

We refer to a "linear manifold" to include the case where $S$ has infinite dimension. For instance, we can construct an infinite dimensional complex symplectic space $S=H_{-} \oplus H_{+}$, as the direct sum of two Hilbert spaces $H_{-}$and $H_{+}$, so each vector $u \in S$ has a unique representation $u=u_{-}+u_{+}$with $u_{ \pm} \in H_{ \pm}$, respectively. We take the sympectic form

$$
[u: v]=\left[u_{-}+u_{+}: v_{-}+v_{+}\right]=-i\left\langle u_{-}, v_{-}\right\rangle_{-}+i\left\langle u_{+}, v_{+}\right\rangle_{+}
$$

in terms of the Hermitian inner products $\langle\cdot, \cdot\rangle_{ \pm}$in $H_{ \pm}$, respectively (compare the corresponding finite dimensional cases in Theorem 3 below).

However, in this paper we deal only with complex symplectic spaces $S$ of finite (complex) dimension $D \geq 0$ (the case $D=0$ defines the trivial space consisting of just a single point, and this case is often omitted in the subsequent discussions), and then each linear submanifold is a linear subspace of $S$ (that is, closed in the usual topology of $S$, as in $\mathbf{C}^{D}$ ).

In the following Example 1, we note that each complex symplectic space $S$ with finite dimension $D \geq 1$ is isomorphic to the complex number space $\mathbf{C}^{D}$ with a suitable complex symplectic structure - that is, with a symplectic form on the complex vector space $\mathbf{C}^{D}$.

Example 1. Skew-Hermitian matrices and complex symplectic spaces in $\mathbf{C}^{D}$.

Let $S$ with the symplectic form [:] be a complex symplectic space of finite dimension $D \geq 1$. Then there exists a linear bijective map of $S$ onto the complex number space $\mathbf{C}^{D}$, and accordingly the symplectic products in $S$ can be expressed in terms of the complex coordinates that are induced by this map, say 
$u=\left(u_{1}, u_{2}, \ldots, u_{D}\right), v=\left(v_{1}, v_{2}, \ldots, v_{D}\right)$ for (row) vectors defining $u, v \in S$. Namely, write the sesquilinear form on $\mathbf{C}^{D}$ as

$$
[u: v]=\left(u_{1}, \ldots, u_{D}\right) H\left(v_{1}, \ldots, v_{D}\right)^{*},
$$

where $H$ is the corresponding $D \times D$ skew-Hermitian matrix, and hence $H$ satisfies the conditions:

$$
\begin{aligned}
& H=-H^{*} \text { (here } H^{*}=\bar{H}^{t}, \text { where }{ }^{*} \text { denotes the conjugate transpose), } \\
& \operatorname{det} H \neq 0 \text {, so } H \text { is nonsingular. }
\end{aligned}
$$

These conditions (1.6) are merely the axioms (1.1) (i) (ii) (iii) interpreted in matrix notation. Hence each skew-Hermitian non-singular $D \times D$ matrix $H$ defines a complex symplectic product (1.5) on $\mathbf{C}^{D}$, and moreover each complex symplectic $D$-space $S$ is isomorphic to such a complex symplectic $\mathbf{C}^{D}$. That is, the most general complex symplectic $D$-space $S$ has an isomorphism onto $\mathbf{C}^{D}$, once a basis is chosen for $S$, and then the symplectic products are specified by a skew-Hermitian nonsingular $D \times D$ matrix $H$, as in (1.5) or

$$
[u, v]=u H v^{*} \text {. }
$$

However, a linear change of basis in $S$ yields new numerical coordinates, say $u$ has the components

$$
\tilde{u}=\left(\tilde{u}_{1}, \ldots, \tilde{u}_{D}\right) \text { where } u_{j}=\tilde{u}_{k} Q_{j}^{k}
$$

for a complex non-singular matrix $Q=\left(Q_{j}^{k}\right)$. Then

$$
[u: v]=(\tilde{u} Q) H(\tilde{v} Q)^{*}=\tilde{u} \tilde{H} \tilde{v}^{*},
$$

in terms of the congruent matrix $\tilde{H}=Q H Q^{*}$. Since $(i H)^{*}=-i H^{*}=i H$ is symmetric Hermitian, there exists some complex non-singular matrix $Q$ such that $Q(i H) Q^{*}=\operatorname{diag}\left\{I_{q},-I_{p}\right\}$, so

$$
\tilde{H}=\operatorname{diag}\left\{-i I_{q} i I_{p}\right\}, \quad\left(i^{2}=-1\right) .
$$

Here $I_{q}$ is the identity matrix of size $q \geq 0$ (omitted when $q=0$ ), and similarly for $I_{p}$ of size $p \geq 0$. This yields an important diagonal format (1.8) for the skewHermitian non-singular $D \times D$ matrix $H$, with $p+q=D$.

As an interesting special example take $D=3$, and consider the complex linear space $S=\mathbf{C}^{3}$ with the prescribed symplectic products $\left[e^{1}: e^{1}\right]=i,\left[e^{2}: e^{2}\right]=$ $i,\left[e^{3}: e^{3}\right]=-i$, and all other symplectic products are zero for the customary basis vectors

$$
e^{1}=(1,0,0), e^{2}=(0,1,0), e^{3}=(0,0,1) .
$$

That is, we use the skew-Hermitian matrix $H=\operatorname{diag}\{i, i,-i\}$ to define the symplectic structure on $\mathbf{C}^{3}$.

In this complex symplectic 3 -space $S$ the 1-dimensional subspace

$$
L=\operatorname{span}\left\{e^{2}+e^{3}\right\}=\{(0, c, c)\}, \quad \text { for all } c \in \mathbf{C},
$$

can easily be verified to be Lagrangian since $\left[e^{2}+e^{3}: e^{2}+e^{3}\right]=i-i=0$. It will be shown later, see Theorem 1 below, that there are no Lagrangian 2-spaces in $S$; but $L$ is not a complete Lagrangian subspace, since $\left[e^{1}: L\right]=0$ yet $e^{1} \notin L$.

Example 2. Real symplectic spaces of Lagrangian classical dynamics, and canonical bases. 
A real symplectic space $S_{R}$ is a real linear space, together with a prescribed real symplectic form $[:]_{R}$, namely a real bilinear form

$$
u, v \rightarrow[u: v]_{R}, \quad S_{R} \times S_{R} \rightarrow \mathbf{R},
$$

which is skew-symmetric,

$$
[u: v]_{R}=-[v: u]_{R},
$$

and also non-degenerate or non-singular,

$$
\left[u: S_{R}\right]=0 \text { implies } u=0,
$$

for all vectors $u, v \in S_{R}$.

Following the earlier Definition 2 above, we define a linear manifold $L \subset S_{R}$ to be Lagrangian in case $[L: L]_{R}=0$, and, in addition, $L$ is complete just in case

$$
u \in S_{R} \text { and }[u: L]_{R}=0 \text { imply } u \in L .
$$

We remark that in much of the literature on real symplectic spaces, only complete Lagrangian submanifolds of $S_{R}$ are referred to as "Lagrangian" (the others are termed "isotropic"), see [AM], [MH], [MS].

Just as in Example 1 we note that a real symplectic space $S_{R}$ of finite (real) dimension $D \geq 1$ is symplectically isomorphic with $\mathbf{R}^{D}$ bearing a suitable bilinear form

$$
[u: v]_{R}=u A v^{t}, \text { with } A=-A^{t}, \operatorname{det} A \neq 0 .
$$

But a real skew-symmetric non-singular matrix $A$ must be of even size $D=2 n$, and is necessarily congruent (via a real non-singular matrix $Q$ ) to the canonical matrix

$$
Q A Q^{t}=\left(\begin{array}{cc}
0 & I_{n} \\
-I_{n} & 0
\end{array}\right) .
$$

A corresponding basis that achieves this canonical matrix (1.10) is called a canonical basis, say

$$
\left\{e^{1}, e^{2}, \ldots, e^{n}, e^{n+1}, e^{n+2}, \ldots, e^{2 n}\right\} \quad \text { in } S_{R}\left(\text { or in } \mathbf{R}^{2 n}\right),
$$

with the corresponding symplectic products

$$
\left[e^{j}: e^{k}\right]_{R}=0,\left[e^{n+j}: e^{n+k}\right]_{R}=0, \text { for } 1 \leq j, k \leq n,
$$

and the familiar canonical duality (or canonical conjugation)

$$
\left[e^{j}: e^{n+k}\right]=\delta^{j k} \quad(\text { Kronecker } \delta) .
$$

Then we recognize that, for $\operatorname{dim} S_{R}=2 n$,

$$
\operatorname{span}\left\{e^{1}, \ldots, e^{n}\right\} \text { and } \operatorname{span}\left\{e^{n+1}, \ldots, e^{2 n}\right\}
$$

are each complete Lagrangian subspaces in $S_{R}$, and moreover are related through the canonical conjugation (1.13).

The existence of such canonical bases in $S_{R}$ shows that there is a unique (up to symplectic isomorphism) real symplectic space of dimension $2 n$, and we often denote this symplectic space as $\mathbf{R}^{2 n}$. It is known that a Lagrangian subspace $L$ of $\mathbf{R}^{2 n}$ is complete if and only if $\operatorname{dim} L=n$, see [AM], [MA], [MH].

These concepts originated in the Lagrangian classical dynamics of physical systems with $n$ degrees of freedom, where the generalized coordinates consist of $n$ generalized positions, corresponding to $\left\{e^{1}, \ldots, e^{n}\right\}$, and $n$ generalized momenta, corresponding to $\left\{e^{n+1}, \ldots, e^{2 n}\right\}$. 
It is easy to complexify a real symplectic space $S_{R}$ so as to construct a complex symplectic space $S$ by simply using complex vectors. More precisely, $S$ consists of all ordered pairs $\{X, Y\}$ of vectors of $S_{R}$, with the obvious algebraic operations suggested by the familiar notation

$$
Z=X+i Y, \text { for } X, Y \in S_{R} .
$$

Namely, use componentwise vector addition in $S$; also multiplication by complex scalars $\mu=\alpha+i \beta$ (for $\alpha, \beta \in \mathbf{R}$ ) in accord with

$$
\mu Z=(\alpha+i \beta)(X+i Y)=(\alpha X-\beta Y)+i(\beta X+\alpha Y),
$$

and define the symplectic product in $S$ by

$$
\begin{aligned}
{\left[Z_{1}: Z_{2}\right] } & =\left[X_{1}+i Y_{1}: X_{2}+i Y_{2}\right] \\
& =\left[X_{1}: X_{2}\right]_{R}+\left[Y_{1}: Y_{2}\right]_{R}+i\left\{\left[Y_{1}: X_{2}\right]_{R}+\left[Y_{2}: X_{1}\right]_{R}\right\} .
\end{aligned}
$$

Further, we note that there is an involutary bijection on $S$, called complex conjugation,

$$
Z=X+i Y \rightarrow \bar{Z}=X-i Y
$$

so then

$$
\left.\overline{\bar{Z}}_{1}=Z_{1}, \quad \overline{\mu_{1} Z_{1}+\mu_{2} Z_{2}}=\bar{\mu}_{1} \bar{Z}_{1}+\bar{\mu}_{2} \bar{Z}_{2}, \quad \overline{\left[Z_{1}: Z_{2}\right.}\right]=\left[\bar{Z}_{1}: \bar{Z}_{2}\right],
$$

and the properties (1.14), (1.15), (1.16), (1.17) hold for all vectors $Z_{1}=X_{1}+i Y_{1}$, $Z_{2}=X_{2}+i Y_{2}$ in $S$, and complex scalars $\mu_{1}, \mu_{2} \in \mathbf{C}$. Moreover, the "real vectors" of $S$, namely $X+i O$, defined as the invariant or fixed vectors under the complex conjugation, constitute a subset $S_{R}^{\prime} \subset S$ which is itself a real symplectic space with respect to the algebraic operations induced from $S$-using real vectors and real scalars. Clearly $S_{R}^{\prime}$ contains all vectors

$$
X=\operatorname{Re} Z=\frac{1}{2}(Z+\bar{Z}), \quad Y=\operatorname{Im} Z=\frac{1}{2 i}(Z-\bar{Z}),
$$

and it is immediate to verify that $S_{R}^{\prime}$ and $S_{R}$ are isomorphic real symplectic spaces under the map

$$
X+i O \rightarrow X .
$$

A basis for $S_{R}^{\prime}$ (over the real scalars) serves also as a basis for $S$ (over the complex scalars). In particular, a canonical basis for $S$ consists of a canonical basis for $S_{R}^{\prime}$-for which the corresponding skew-Hermitian matrix is given in (1.10).

It follows that

$$
\text { (real) } \operatorname{dim} S_{R}^{\prime}=\text { (complex) } \operatorname{dim} S
$$

(if either is infinite, so is the other).

In any case the complex symplectic space $S$, with its distinctive complex conjugation, is the unique such space (up to isomorphism in the category of complex symplectic spaces with complex conjugation) for which $S_{R}^{\prime}$ is isomorphic to $S_{R}$ (as real symplectic spaces). In this sense we are entitled to refer to the unique complexification $S$ of the real symplectic space $S_{R}$; see [EM] for further details.

As a final remark on this topic, note that the complex symplectic space $\mathbf{C}^{3}$ of Example 1 above is not the complexification of any real symplectic space. Furthermore, as will be obvious from Theorem 1 below, there are many non-isomorphic symplectic structures on each complex vector space $\mathbf{C}^{D}$ for $D \geq 1$, such that $\mathbf{C}^{D}$ then becomes a complex symplectic space which is not the complexification of any 
real symplectic space. In this sense, the category of complex symplectic spaces is a non-trivial generalization of that of real symplectic spaces.

In the Appendix at the close of this paper we apply the theory of complex symplectic spaces to the boundary value problems of general (formally self-adjoint) linear differential operators of arbitrary orders $n \geq 1$, with complex coefficients defined on arbitrary real intervals (open, closed, half-closed, finite, or infinite). This is accomplished by the famous result of Glazman-Krein-Naimark, the GKNtheorem [EM], [EM1], [GZ], which specifies a natural one-to-one correspondence between the set of all self-adjoint boundary conditions, and the set of all complete Lagrangian subspaces of the endpoint complex symplectic space for the differential operator, as explained in Example 3 below. A new and self-contained proof of the GKN-theorem is presented in the Appendix.

However, in the next Example 3 we merely sketch the motivation for these ideas, as illustrated by the classical Sturm-Liouville differential operator on a compact interval - that is, by the most elementary and familiar "regular boundary value problem".

Example 3. Illustrations for regular boundary value problems: the GKN-theorem.

Consider the Sturm-Liouville second-order differential operator or expression

$$
M[y](x) \equiv-\left(p(x) y^{\prime}(x)\right)^{\prime}+q(x) y(x), \quad \text { for all } x \in \mathcal{I}=[0,1],
$$

where $p, q: \mathcal{I} \rightarrow \mathbf{R}$ are suitably smooth real coefficients, and $p(x)$ is nowhere zero on $\mathcal{I}$ (say, $p \in A C(\mathcal{I})$, absolutely continuous, and $q \in L^{1}(\mathcal{I})$, Lebesgue integrable on the compact interval $\mathcal{I}$, in the usual notation). See [EM], [EZ] for further details for this Example 3.

In the study of the boundary value problem for the differential expression $M$ on the compact real interval $\mathcal{I}$ (notably, a Lagrange-symmetric or formally self-adjoint differential expression), we investigate the eigenvalues $\lambda \in \mathbf{C}$ for

$$
M[y] \equiv-\left(p y^{\prime}\right)^{\prime}+q y=\lambda y,
$$

where $y$ is to be restricted (by boundary conditions at the endpoints of $\mathcal{I}$ ) to some specified linear submanifold $D(T)$ of functions:

$$
D(T) \subseteq D_{\max }(M) \subset L^{2}(\mathcal{I}),
$$

whereon $M$ generates the unbounded operator $T$, that is,

$$
T[y]=M[y] \text { for } y \in D(T) .
$$

Here $L^{2}(\mathcal{I})$ denotes the usual Hilbert space of all complex-valued square-integrable functions (or appropriate equivalence classes of functions) on $\mathcal{I}$, with the Hermitian inner (or scalar) product:

$$
\langle f, g\rangle=\int_{0}^{1} f \bar{g} d x, \text { for } f, g \in L^{2}(\mathcal{I}) .
$$

Further, we define the maximal domain for $M$ (as an operator in $L^{2}(\mathcal{I})$ ),

$$
D_{\text {max }}(M)=\left\{f: \mathcal{I} \rightarrow \mathbf{C} \mid f, f^{\prime} \in A C(\mathcal{I}) \text { and } M[f] \in L^{2}(\mathcal{I})\right\},
$$

and also the minimal domain for $M$,

$$
D_{\min }(M)=\left\{f \in D_{\max }(M) \mid f(0)=f^{\prime}(0)=f(1)=f^{\prime}(1)=0\right\},
$$


on which $M$ generates the corresponding maximal and minimal operators, respectively,

$$
T_{\max } \text { on } D\left(T_{\max }\right) \equiv D_{\max }(M) \text { and } T_{\min } \text { on } D\left(T_{\min }\right) \equiv D_{\min }(M) .
$$

It is known [DS], [EZ], [NA] that $T_{\min }$ and $T_{\max }$ are closed linear operators, with dense domains in $L^{2}(\mathcal{I})$, and with adjoint operators:

$$
T_{\min }^{*}=T_{\max } \text { and } T_{\max }^{*}=T_{\min } .
$$

We seek domains $D(T) \subseteq D_{\max }(M)$ on which $M$ generates a self-adjoint operator $T$, that is,

$$
T^{*}=T \text { on } D\left(T^{*}\right)=D(T) .
$$

For such self-adjoint operators $T$ it follows from the basic properties of adjoints that

$$
T_{\min } \subseteq T \subseteq T_{\max } \quad \text { on } \quad D_{\min }(M) \subseteq D(T) \subseteq D_{\max }(M) .
$$

In this situation, the eigenvalues of $T$, and all its spectral properties, are of signal importance in both pure and applied mathematics - especially with reference to functional analysis. As mentioned earlier, the GKN-theorem, which is proved in the Appendix, demonstrates a natural one-to-one correspondence between the set of all self-adjoint operators $T$, as generated by $M$ on $D(T)$, and the set of all complete Lagrangian subspaces $\mathbf{L}$ of the endpoint complex symplectic space $\mathbf{S}$ for $M$ on $\mathcal{I}$.

We now define the complex vector space (endpoint space for $M$ on $\mathcal{I}$ )

$$
\mathbf{S}:=D_{\max }(M) / D_{\min }(M),
$$

the quotient or identification space, so each element $\mathbf{f}$ is a coset $\mathbf{f}=\left\{f+D_{\min }(M)\right\}$ of some function $f \in D_{\max }(M)$.

Note. The bold-face notation for $\mathbf{f}, \mathbf{L}$ and $\mathbf{S}$ will be used here (and in the Appendix) to emphasize that these are cosets, or collections of cosets, in the identification space of functions $f \in D_{\max }(M)$, as in (1.31).

Then we define the symplectic product of $\mathbf{f}, \mathbf{g} \in \mathbf{S}$ by

$$
[\mathbf{f}: \mathbf{g}]=\left[f+D_{\min }(M): g+D_{\min }(M)\right]:=[f: g],
$$

where the skew-Hermitian form $[f: g]$ is defined for $f, g \in D_{\max }(M)$ by

$$
[f: g]=\langle M[f], g\rangle-\langle f, M[g]\rangle=\left[-p f^{\prime} \bar{g}+f p \bar{g}^{\prime}\right]_{x=0}^{1} .
$$

We note that (see the Appendix and the monograph $[\mathrm{EM}]$ )

$$
D_{\min }(M)=\left\{f \in D_{\max }(M) \mid\left[f: D_{\max }(M)\right]=0\right\},
$$

so $T_{\min }$ is a symmetric (but not necessarily self-adjoint) operator; and, accordingly, the symplectic form [:] is well-defined on $\mathbf{S}$ and also is appropriately non-degenerate in the sense of Definition 1. Hence the endpoint space $\mathbf{S}$ is the desired complex symplectic space. In this case, it is easily demonstrated that $\operatorname{dim} \mathbf{S}=4$, see (1.26).

Further, we can construct the left and right endpoint spaces for $M$ on $\mathcal{I}$, respectively, by

$$
\mathbf{S}_{-}=\left\{\mathbf{f} \in \mathbf{S} \mid f(1)=f^{\prime}(1)=0\right\}, \quad \mathbf{S}_{+}=\left\{\mathbf{f} \in \mathbf{S} \mid f(0)=f^{\prime}(0)=0\right\} .
$$

These establish a "symplectic orthogonal" direct sum decomposition for $\mathbf{S}$,

$$
\mathbf{S}=\mathbf{S}_{-} \oplus \mathbf{S}_{+} \text {, with }\left[\mathbf{S}_{-}: \mathbf{S}_{+}\right]=0,
$$


providing useful methods for classifying the Lagrangian subspaces $\mathbf{L} \subset \mathbf{S}$, say:

(1.37)

strictly separated boundary conditions : $\operatorname{dim} L=\operatorname{dim} L \cap \mathbf{S}_{-}+\operatorname{dim} L \cap \mathbf{S}_{+}$,

totally coupled boundary conditions : $\operatorname{dim} L \cap \mathbf{S}_{-}=\operatorname{dim} L \cap \mathbf{S}_{+}=0$.

From (1.26) we see that $\operatorname{dim} \mathbf{S}=4$, so the complex symplectic space $\mathbf{S}$ is linearly isomorphic to $\mathbf{C}^{4}$, and we can introduce corresponding coordinates in $\mathbf{S}$ by the convenient choice

$$
\mathbf{f}=\left(f(0),\left(p f^{\prime}\right)(0), f(1),\left(p f^{\prime}\right)(1)\right),
$$

where $\mathbf{f}=\left\{f+D_{\min }(M)\right\}$, for $f \in D_{\max }(M)$, and we recall that $p(0) \neq 0, p(1) \neq 0$.

Next introduce the corresponding symplectic product in $\mathbf{C}^{4}$ using the skewHermitian $4 \times 4$ matrix

$$
H=\left(\begin{array}{rrrr}
0 & -1 & 0 & 0 \\
1 & 0 & 0 & 0 \\
0 & 0 & 0 & 1 \\
0 & 0 & -1 & 0
\end{array}\right),
$$

so the symplectic product in $\mathbf{S}$ can be expressed in terms of these coordinates, see (1.33) and (1.38), by

$$
[\mathbf{f}: \mathbf{g}]=\mathbf{f} H \mathbf{g}^{*} \text {. }
$$

Thus the boundary value problem for the Sturm-Liouville expression $M$ on $\mathcal{I}=[0,1]$ is reduced, via the GKN-theorem to the purely algebraic problem of determining all the complete Lagrangian subspaces $\mathbf{L}$ in this complex symplectic space $\mathbf{C}^{4}$. By Theorem 2 below, $\mathbf{L} \subset \mathbf{S}$ is complete if and only if $\operatorname{dim} \mathbf{L}=2$. For example, use the customary unit basis vectors in $\mathbf{C}^{4}$,

$$
e^{1}=(1,0,0,0), e^{2}=(0,1,0,0), e^{3}=(0,0,1,0), \text { and } e^{4}=(0,0,0,1),
$$

so $\mathbf{f}=f(0) e^{1}+\left(p f^{\prime}\right)(0) e^{2}+f(1) e^{3}+\left(p f^{\prime}\right)(1) e^{4}$, and define the Lagrangian 2-space

$$
\mathbf{L}_{s}=\operatorname{span}\left\{e^{2}, e^{4}\right\},
$$

which is a complete Lagrangian subspace of $\mathbf{C}^{4}$, or of $\mathbf{S}$ by virtue of the symplectic isomorphism of $\mathbf{S}$ with $\mathbf{C}^{4}$. Namely, $\mathbf{L}_{s}$ is the subspace specified by the null space of the linear functionals $f(0)$ and $f(1)$, which we customarily denote by the two boundary conditions

$$
f(0)=0, f(1)=0 \quad(\text { strictly separated, see (1.37)). }
$$

Next take $f_{1}, f_{2} \in D_{\max }(M)$ satisfying these boundary conditions, and such that $\left\{\mathbf{f}_{1}, \mathbf{f}_{2}\right\}$ constitutes a basis for $\mathbf{L}_{s}$. Then the domain $D(T)$ of the corresponding self-adjoint operator $T$ in $L^{2}(\mathcal{I})$ is given by the GKN-theorem to be

$$
D(T)=c_{1} f_{1}+c_{2} f_{2}+D_{\min }(M),
$$

for arbitrary numbers $c_{1}, c_{2} \in \mathbf{C}$.

Similarly, another complete Lagrangian is defined by

$$
\mathbf{L}_{c}=\operatorname{span}\left\{e^{1}+e^{3}, e^{2}+e^{4}\right\},
$$

given traditionally by the boundary conditions (compare notation of quasi-derivatives, see $[\mathrm{EV}])$

$$
f(0)=f(1), p(0) f^{\prime}(0)=p(1) f^{\prime}(1) \quad(\text { totally coupled }(1.37)) .
$$


For a general (formally self-adjoint i.e. Lagrange symmetric, see [DS], [ER]) linear differential expression $M$ of arbitrary order $n \geq 1$, with complex coefficients on an arbitrary real interval $\mathcal{J}$ (finite or infinite), the maximal and minimal operators

$$
T_{\max } \text { on } D_{\max }(M) \text { and } T_{\min } \text { on } D_{\min }(M) \text {, }
$$

and the endpoint complex symmplectic space

$$
\mathbf{S}=D_{\max }(M) / D_{\min }(M)
$$

with the symplectic form inherited from $D_{\max }(M)$, as in (1.32),

$$
[\mathbf{f}: \mathbf{g}]=[f: g]=\langle M[f], g\rangle-\langle f, M[g]\rangle,
$$

are all constructed just as in Example 3, but with obvious minor modifications; see our Appendix. Moreover, $\operatorname{dim} \mathbf{S} \leq 2 n$ (with equality always holding in the regular case where $\mathcal{J}$ is compact) and by Theorem 2 we need only consider the case where $\operatorname{dim} \mathbf{S}=2 d$ is even.

Within this general framework the Glazman-Krein-Naimark (GKN) Theorem obtains:

There exists a natural one-to-one correspondence between the set of all selfadjoint operators

$$
T \text { on } D(T) \subseteq D_{\max }(M) \subset L^{2}(\mathcal{J}),
$$

as generated by $M$ on $\mathcal{J}$, and the set of all complete Lagrangian subspaces $\mathbf{L} \subset \mathbf{S}$.

In particular, for each complete Lagrangian $\mathbf{L} \subset \mathbf{S}$ with $\operatorname{dim} \mathbf{L}=d$, let $f_{1}, \ldots, f_{d}$ in $D_{\max }(M)$ be such that $\left\{\mathbf{f}_{1}, \ldots, \mathbf{f}_{d}\right\}$ constitutes a basis for $\mathbf{L}$. Then the corresponding domain $D(T)$ for the self-adjoint operator $T$ is

$$
D(T)=c_{1} f_{1}+\cdots+c_{d} f_{d}+D_{\min }(M),
$$

where $c_{1}, \ldots, c_{d}$ are arbitrary complex numbers.

A precise statement of the GKN-theorem, and a new proof, are presented in the Appendix to this paper.

\section{Finite dimensional Complex symplectic spaces, AND THEIR LAGRANGIAN SUBSPACES}

We consider the algebra of complex symplectic spaces $S$ of finite dimension $D \geq 0$. While many of these results hold also for infinite dimensional complex symplectic spaces, we defer that development to some later work.

The first theorem in this section analyses various symplectic invariants that characterize $S$, up to symplectic isomorphism. The next theorem treats the positioning of a Lagrangian subspace within $S$, and gives necessary and sufficient conditions for the existence of complete Lagrangian subspaces. The final three theorems deal with various "symplectic orthogonal decompositions" of $S$ which arise in the applications to boundary value problems for linear differential operators (although the methods are strictly algebraic).

Accordingly, take a complex symplectic space $S$ of finite dimension $D \geq 1$ (the case $D=0$ is the trivial space consisting of a single point, and will often be omitted from the discussions). It is evident that in such a complex symplectic space $S$, with symplectic form [:], each vector $v \in S$ satisfies $\operatorname{Re}[v: v]=0$, and hence $v$ (in fact, 
each 1-dimensional subspace $\mu v$ for $\mu \in \mathbf{C}$, where $[\mu v: \mu v]=\mu \bar{\mu}[v: v])$ is of exactly one of the following three types:

$$
\begin{array}{ll}
\text { (i) positive, } & \operatorname{Im}[v: v]>0, \\
\text { (ii) negative, } & \operatorname{Im}[v: v]<0, \\
\text { (iii) neutral, } & \operatorname{Im}[v: v]=0, \text { so }[v: v]=0 .
\end{array}
$$

A Lagrangian subspace $L \subset S$ consists of neutral vectors, so that $[u: v]=0$ for all $u, v \in L$.

We shall use these ideas to obtain invariants for complex symplectic $D$-spaces, that is, we shall consider $\mathbf{C}^{D}$ with an arbitrary complex symplectic form [:].

Definition 1. In a complex symplectic space $S$ with form [:], and finite dimension $D \geq 1$, define the following symplectic invariants of $S$ :

$$
\begin{aligned}
& p=\max \{\text { complex dimension of linear subspaces whereon } \operatorname{Im}[v: v] \geq 0\}, \\
& q=\max \{\text { complex dimension of linear subspaces whereon } \operatorname{Im}[v: v] \leq 0\},
\end{aligned}
$$

$(p, q)$ is called the signature of $S$, consisting of the pair of integers: the positivity index $p \geq 0$ and the negativity index $q \geq 0$.

In addition, we define the Lagrangian index $\Delta$ of $S$,

$$
\Delta=\max \{\text { complex dimension of Lagrangian subspaces of } S\},
$$

and also the excess of $S$,

$$
E x=p-q, \quad \text { excess of positivity over negativity indices of } S .
$$

These symplectic invariants of $S$,

$$
p, q,(p, q), E x, \Delta,
$$

are each defined intrinsically in terms of the symplectic structure on $S$. But in the next Theorem 1 we relate these invariants to the standard diagonal format of the matrix $H$, which defines the corresponding symplectic form in $\mathbf{C}^{D}$, or in $S$ relative to some basis. The important situation, where $D=2 \Delta$, occurs in the GKNTheorem mentioned in the introductory Section 1, and so this case is emphasized in Theorem 1 below and in applications to the theory of linear differential operators.

Theorem 1. Consider a complex symplectic space $S$, with symplectic form [:], and finite dimension $D \geq 1$. Choose any basis on $S$, with corresponding coordinates and skew-Hermitian nonsingular matrix $H$ determined by [:], and hence $H$ is congruent to some diagonal matrix of format $\operatorname{diag}\{i, i, \ldots, i,-i,-i, \ldots,-i\}$. Then conclude that the symplectic invariants (2.2) of $S$ are related to $H$ by:

$$
\begin{aligned}
& p=\text { number of }(+i) \text { terms on the diagonal, } \\
& q=\text { number of }(-i) \text { terms on the diagonal, }
\end{aligned}
$$

with $0 \leq p, q \leq D$, so the diagonal format for $H$ is unique. Also, see (2.3), (2.4),

$$
\begin{aligned}
D= & p+q, \\
E x= & p-q, \\
\Delta= & \min \{p, q\}=\frac{1}{2}(D-|E x|) \leq \frac{1}{2} D, \\
& \text { with equality if and only if } E x=0 .
\end{aligned}
$$


Furthermore, each of the following pairs of invariants is complete and thus characterizes $S$, up to complex symplectic isomorphism:

$$
(p, q) \text { or }(D, E x) \text { or }(\Delta, E x) .
$$

In particular, $S$ is the complexification of the unique real symplectic space $\mathbf{R}^{D}$ if and only if any one of the following logically equivalent conditions obtains:

(i) $D=2 p \quad$ (so $D$ is even),

(ii) $p=q$,

(iii) $E x=0$,

(iv) $D=2 \Delta$,

(v) there exist bases in $S$ for which the skew-Hermitian nonsingular matrix $H$ of the symplectic form becomes

$$
K=\left(\begin{array}{cc}
0 & I_{p} \\
-I_{p} & 0
\end{array}\right) \text { (so } p=q \text {, and the basis is thereby canonical, }
$$
see Section 1 (1.10) and Example 2),

or equally well

$$
\hat{K}=\left(\begin{array}{cc}
i I_{p} & 0 \\
0 & -i I_{p}
\end{array}\right) \quad(\text { so } p=q)
$$

or equally well

$$
J=i^{p} \operatorname{diag}\left(J_{p},-J_{p}\right) \text { with } J_{p}=\left(\begin{array}{ccccc}
0 & 0 & \ldots & 0 & (-1)^{p} \\
0 & 0 & \ldots & \cdot & 0 \\
\vdots & . & . & & \vdots \\
0 & (-1)^{2} & & 0 & 0 \\
(-1) & 0 & \cdots & 0 & 0
\end{array}\right) \quad(\text { so } p=q)
$$

Proof. The complex symplectic space $S$ is linearly isomorphic with $\mathbf{C}^{D}$, and we can choose a basis and corresponding complex coordinates in $S$ so that the symplectic product of vectors $u, v \in S$ is computed by

$$
[u: v]=\left(u_{1}, \ldots, u_{D}\right) \hat{K}\left(v_{1}, \ldots, v_{D}\right)^{*},
$$

where

$$
\hat{K}=\operatorname{diag}\{i, i, \ldots, i,-i,-i, \ldots,-i\}
$$

with $\pi \geq 0$ terms $(+i)$ and $\nu \geq 0$ terms $(-i)$. Of course, some such diagonal matrix $\hat{K}$ is congruent to $H$, and we seek to prove that

$$
p=\pi \quad \text { and } \quad q=\nu .
$$

Consider the linear subspace $P_{+} \subset S$ spanned by the first $\pi$ vectors of the basis for $S$, so $\operatorname{dim} P_{+}=\pi$. But $\operatorname{Im}[v: v] \geq 0$ for all $v \in P_{+}$, so $p \geq \pi$. We shall show that for each linear subspace $P \subset S$ whereon $\operatorname{Im}[v: v] \geq 0$ we have $\operatorname{dim} P \leq \pi$, which will demonstrate that $p=\pi$.

Consider the subspace $N_{-} \subset S$ spanned by the last $\nu$ vectors of the basis for $S$, so $\operatorname{dim} N_{-}=\nu$. But note that $\operatorname{Im}[v: v]<0$ for every non-zero vector $v \in N_{-}$, and hence that $P \cap N_{-}=\{0\}$. This implies that $\operatorname{dim} P+\nu \leq D$, and hence $\operatorname{dim} P \leq D-\nu=\pi$. 
We have proved that $p=\pi$, and a similar argument shows that $q=\nu$, for each such diagonal matrix $\hat{K}$ congruent to the skew-Hermitian nonsingular matrix $H$. In this sense the diagonal format $\hat{K}$ for matrices expressing the symplectic form of $S$ is unique (except possibly for the ordering of the diagonal elements), since $p$ and $q$ are symplectic invariants of $S$, as in (2.2).

It is now clear that the signature $(p, q)$ determines $S$ with [:], up to symplectic isomorphism. Hence the pair $(D, E x)$, where $D=p+q$ and $E x=p-q$, also characterizes this complex symplectic space.

We next examine the symplectic invariant $\Delta$ (see (2.3)), and relate it to the signature $(p, q)$ of the complex symplectic space $S$. For definiteness take the case $p \geq q$, so the excess $E x \geq 0$. Choose a basis for $S$ (merely a rearrangement of the previous basis) so that the matrix of the symplectic form for $S$ becomes

$$
\left(\begin{array}{ccc}
i I_{q} & 0 & 0 \\
0 & -i I_{q} & 0 \\
0 & 0 & i I_{E x}
\end{array}\right) .
$$

That is, $S$ is then the direct sum of three subspaces of dimensions $q, q$, and $E x$ (omit $i I_{E x}$ if $E x=0$ ). Let this chosen basis be indicated by $q+q+E x$ vectors

$$
\left\{e^{1}, e^{2}, \ldots, e^{q}, f^{1}, f^{2}, \ldots, f^{q}, g^{1}, \ldots, g^{E x}\right\},
$$

where $\left\{e^{1}, \ldots, e^{q}, f^{1}, \ldots, f^{q}\right\}$ span a symplectic subspace of $S$-which we denote by $\mathbf{C}^{2 q}$ - having dimension $2 q$ and excess zero.

Within this symplectic subspace $\mathbf{C}^{2 q}$ define the Lagrangian subspace $L_{1}$,

$$
L_{1}=\operatorname{span}\left\{e^{1}+f^{1}, e^{2}+f^{2}, \ldots, e^{q}+f^{q}\right\} .
$$

Since $\operatorname{dim} L_{1}=q$ we conclude that $\Delta \geq q$.

Now note that there are $p=q+E x$ independent vectors in $S$, which span $P_{+}$ whereon $\operatorname{Im}[v: v]>0$ (except for $v=0$ ). Thus each Lagrangian subspace $L \subset S$ can meet $P_{+}$only at the origin. Hence $\operatorname{dim} L+p \leq D$, so $\operatorname{dim} L \leq D-p=q$. Therefore $\Delta=q$.

When $q \geq p$ a similar argument applies to prove $\Delta=p$. Therefore, in all cases

$$
\Delta=\min \{p, q\} \text {. }
$$

The remaining relation

$$
\Delta=(D-|E x|) / 2 \leq D / 2
$$

follows directly from an inspection of the diagonal matrix $\hat{K}$ of the format (2.8) or (2.9).

Now consider the pair of invariants $(\Delta, E x)$ for $S$. But $\Delta=\min \{p, q\}$ and $E x=p-q$ together determine the signature $(p, q)$. For if $E x \geq 0$, then $p=E x+\Delta$, $q=\Delta$; but if $E x<0$, then $p=\Delta, q=|E x|+\Delta$. Hence the pair $(\Delta, E x)$ determines the complex symplectic space $S$, up to symplectic isomorphism.

Finally consider the case where $S$ is symplectically isomorphic to $\mathbf{C}^{2 p}$, assumed to be the complexification of $\mathbf{R}^{2 p}$. In this situation $D=2 p$, so $p=q, E x=0$, and hence $D=2 \Delta$. This follows because there is a real canonical basis for $\mathbf{R}^{2 p}$, relative to which the real symplectic form is defined by the real skew-symmetric matrix $K$ of $(2.7(\mathrm{v}))$,

$$
K=\left(\begin{array}{cc}
0 & I_{p} \\
-I_{p} & 0
\end{array}\right)
$$


But this real canonical basis for $\mathbf{R}^{2 p}$ serves equally well as a canonical basis (over $\mathbf{C}$ ) for the complex symplectic space $\mathbf{C}^{2 p}$. Further, the corresponding skew-Hermitian nonsingular matrix of the symplectic form remains $K$. Moreover, in the complex linear space $\mathbf{C}^{2 p}$ the matrix $K$ is congruent to the skew-Hermitian matrix $\hat{K}$ of $(2.7(\mathrm{v}))$ with $p=q$. Only the equivalence of the condition $(2.7(\mathrm{v}))$, involving the matrix

$$
J=i^{p} \operatorname{diag}\left\{J_{p},-J_{p}\right\},
$$

may cause any concern. However note that when $p$ is even, $J$ is a real skewsymmetric non-singular matrix, and so $J$ is congruent (over $\mathbf{R}$ ) to the matrix $K$. Also when $p$ is odd, then $(i J)$ is a real symmetric matrix with signature $(p, p)$, so $J$ is skew-Hermitian with $p=q$ and $E x=0$. Hence in either case $J$ is congruent (over $\mathbf{C}$ ) to the matrix $K$ of $(2.7(\mathrm{v}))$. Thus we have established that the identities (2.7) hold for $\mathbf{C}^{2 p}$, assuming it is the complexification of the real symplectic space $\mathbf{R}^{2 p}$.

Conversely, consider a complex symplectic space $S$ of dimension $D=p+q=2 p$, see $(2.7(\mathrm{i}))$. Then the remaining identities of (2.7) hold; namely, $p=q, E x=$ $0, D=2 \Delta$, as well as the existence of bases of $S$ so as to yield $K(2.7(\mathrm{v}))$, and hence $\hat{K}$ or $J$. In all cases it is easy to show that any one of the equalities of (2.7) implies all the remaining ones.

So, in this case, fix a canonical basis for $S$, relative to which the skew-symmetric matrix is $K$ of (2.10), and specify these basis vectors as real. Then real linear combinations of these real basis vectors yield the set $S_{R}^{\prime} \subset S$ of real vectors, and these determine the corresponding complex conjugation in $S$. Moreover $S_{R}^{\prime}$ is a real symplectic space isomorphic to $\mathbf{R}^{2 p}$. But $S$ is the complexification of $S_{R}^{\prime}$, and so of $\mathbf{R}^{2 p}$, as required.

Definition 2. Let $S$ be a complex symplectic space with symplectic form [:]. Then linear subspaces (or submanifolds) $S_{-}$and $S_{+}$are symplectic ortho-complements in $S$, written as

$$
S=S_{-} \oplus S_{+},
$$

in case

(i) $S=\operatorname{span}\left\{S_{-}, S_{+}\right\}$,

(ii) $\left[S_{-}: S_{+}\right]=0$.

In this case $S_{-} \cap S_{+}=0$, so $S$ is the direct sum of $S_{-}$and $S_{+}$, that is, each vector $u \in S$ has a unique decomposition $u=u_{-}+u_{+}$with $u_{-} \in S_{-}$and $u_{+} \in S_{+}$. Moreover, $\left[u_{-}: u_{+}\right]=0$ for all $u_{-} \in S_{-}$and $u_{+} \in S_{+}$. From this it follows that

$$
S_{-}=\left\{u \in S \mid\left[u: S_{+}\right]=0\right\} \quad \text { and } \quad S_{+}=\left\{u \in S \mid\left[u: S_{-}\right]=0\right\} .
$$

Furthermore, for such a symplectic orthogonal direct sum decomposition $S=$ $S_{-} \oplus S_{+}$(satisfying conditions (i) and (ii) — and we often also write $\left[S_{-}: S_{+}\right]=0$ explicitly for additional emphasis) each of $S_{-}$and $S_{+}$is itself a complex symplectic space, since the symplectic form induced from [:] is non-degenerate on $S_{-}$and on $S_{+}$.

The next two corollaries of Theorem 1 relate the symplectic invariants of the two symplectic ortho-complements, as in Definition 2, to the invariants of $S$. 
Corollary 1. Consider a complex symplectic space $S$ with symplectic form [:], and finite dimension $D \geq 1$. Then there exists a direct sum representation

$$
S=S^{2 \Delta} \oplus S^{|E x|} \text { with }\left[S^{2 \Delta}: S^{|E x|}\right]=0 .
$$

The complex symplectic subspace $S^{2 \Delta}$ has dimension $2 \Delta$ and excess zero, and the complex symplectic subspace $S^{|E x|}$ has dimension $|E x|$ and excess $p-q$ (in terms of the invariants of $S$ ). Hence $S^{2 \Delta}$ is symplectically isomorphic to the classical complexification of $\mathbf{R}^{2 \Delta}$, and $S^{|E x|}$ contains no non-zero neutral vectors.

If for $S$ the invariant $E x=0$, then $S=S^{2 \Delta}$; but if $\Delta=0$, then $S=S^{|E x|}$.

The subspaces $S^{2 \Delta}$ and $S^{|E x|}$ are not unique in $S$, but are determined only up to symplectic isomorphism.

Proof. This result follows immediately from the diagonal format (2.9) of the skewHermitian matrix

$$
\operatorname{diag}\left\{i I_{\Delta},-i I_{\Delta}, \pm i I_{|E x|}\right\}
$$

(use $+i I_{|E x|}$ when $E x>0$, and $-i I_{|E x|}$ when $E x<0$, and omit this term when $E x=0)$.

The conclusion of Corollary 1 asserts that each complex symplectic space $\mathbf{C}^{D}$ is the direct sum of a complexified $\mathbf{R}^{2 \Delta}$ and a trivial $\mathbf{C}^{|E x|}$ which has no nonzero neutral vectors. We can then choose a relevant basis for $\mathbf{C}^{D}=\mathbf{C}^{2 \Delta} \oplus \mathbf{C}^{|E x|}$, as indicated by the notation

$$
\left\{e^{1}, e^{2}, \ldots, e^{\Delta}, e^{\Delta+1}, \ldots, e^{2 \Delta}, \epsilon^{1}, \ldots, \epsilon^{|E x|}\right\} .
$$

Here the first $2 \Delta$ vectors $\left\{e^{1}, \ldots, e^{\Delta}, e^{\Delta+1}, \ldots, e^{2 \Delta}\right\}$ are a canonical basis for $\mathbf{C}^{2 \Delta}$, and the last $|E x|$ vectors $\left\{\epsilon^{1}, \ldots, \epsilon^{|E x|}\right\}$ are a diagonalizing basis for $\mathbf{C}^{|E x|}$.

Now let us consider a $D$-dimensional complex symplectic space $S$ with [:], and take an arbitrary orthogonal direct sum decomposition into symplectic subspaces $S_{-}$and $S_{+}$as in Definition 2,

$$
S=S_{-} \oplus S_{+} \quad \text { with }\left[S_{-}: S_{+}\right]=0 .
$$

In this situation, there are several relations among the basic invariants, defined in Definition 1 above, namely:

$$
D, p, q, E x \text { and } \Delta \text { for } S,
$$

and the corresponding invariants

$$
D_{ \pm}, p_{ \pm}, q_{ \pm}, E x_{ \pm} \text {and } \Delta_{ \pm}, \quad \text { for } S_{ \pm} \text {, respectively. }
$$

In the next corollary we list some of these relations, which are immediately apparent from an inspection of the diagonalized format of the corresponding skew-Hermitian matrices.

Corollary 2. Let the complex symplectic space S, with symplectic form [:] and finite dimension $D \geq 1$, have a direct sum decomposition as in Definition 2,

$$
S=S_{-} \oplus S_{+} \text {with }\left[S_{-}: S_{+}\right]=0 .
$$

Then the symplectic invariants for $S$ (2.16) and for the symplectic subspaces $S_{ \pm}$ (2.17) satisfy the following conditions:

$$
\begin{aligned}
D & =D_{-}+D_{+}, \quad p=p_{-}+p_{+}, \quad q=q_{-}+q_{+} \\
E x & =E x_{-}+E x_{+}
\end{aligned}
$$


and

$$
\Delta \geq \Delta_{-}+\Delta_{+},
$$

with equality holding if and only if $\left(E x_{-}\right)\left(E x_{+}\right) \geq 0$.

Furthermore, $E x=0$ if and only if $\left(E x_{-}\right)=-\left(E x_{+}\right)$, and in this case

$$
\Delta=\Delta_{-}+\Delta_{+}+\left|E x_{ \pm}\right|,
$$

(note that when $E x=0,\left|E x_{-}\right|=\left|E x_{+}\right|$, which we often denote by $\left|E x_{ \pm}\right|$). If $E x=0$, then $\operatorname{dim} S_{-}=\operatorname{dim} S_{+}$if and only if $\Delta_{-}=\Delta_{+}$.

Proof. Take bases for $S_{-}$and for $S_{+}$, so that the skew-Hermitian matrices of the corresponding symplectic forms are $\hat{K}_{-}$and $\hat{K}_{+}$, respectively, of the diagonal format (2.8). Then the union of these bases constitutes a basis for $S$ with the skewHermitian $D \times D$ matrix

$$
\hat{K}=\operatorname{diag}\left\{\hat{K}_{-}, \hat{K}_{+}\right\} .
$$

Then direct inspection of $\hat{K}$, and elementary arithmetic, yield the required conclusions. For instance, assume $E x=0$ for $S$, and we prove the final assertion of the corollary.

Assume $\operatorname{dim} S_{-}=\operatorname{dim} S_{+}$, and show that $\Delta_{-}=\Delta_{+}$. Without loss of generality we can consider the case

$$
p_{+} \geq q_{+}=\Delta_{+} \text {and } q_{-} \geq p_{-}=\Delta_{-} .
$$

Then, since $\operatorname{dim} S_{+}=\operatorname{dim} S_{-}$and $E x_{+}=-E x_{-}$,

$$
p_{+}+q_{+}=p_{-}+q_{-} \text {and } p_{+}-q_{+}=q_{-}-p_{-} .
$$

Hence

$$
p_{-}=q_{+}, \quad \text { so } \Delta_{-}=\Delta_{+} .
$$

Conversely, assume $\Delta_{-}=\Delta_{+}$. Again take $p_{+} \geq q_{+}=\Delta_{+}$and $q_{-} \geq p_{-}=\Delta_{-}$. But, since $E x_{+}=-E x_{-}$and $p_{+}-q_{+}=q_{-}-p_{-}$, so $p_{+}=q_{-}$. Therefore

$$
p_{+}+q_{+}=q_{-}+p_{-} \quad \text { so } \operatorname{dim} S_{+}=\operatorname{dim} S_{-} \text {. }
$$

Examples of the various cases mentioned in Corollaries 1 and 2 are easily constructed in terms of the corresponding matrices $\hat{K}_{ \pm}$, as introduced in Theorem 1 $(2.8),(2.9)$.

The next Lemma 1 displays the close inter-relations between the symplectic products on $S$, a given finite dimensional complex symplectic space, and the Hermitian inner products on certain ortho-complementary subspaces $N_{-}$and $P_{+}$. The subsequent Lemma 2, and its corollary, use these constructs to demonstrate that the unique symplectic invariant for Lagrangian subspaces $L \subset S$ is the dimension of $L$. These results make elementary the proof of the important Theorem 2 on the existence of complete Lagrangians.

Lemma 1. Consider a complex symplectic space $S$, with symplectic form [:], and finite dimension $D \geq 1$. 
Select a basis for $S$ so that the corresponding skew-Hermitian $D \times D$ matrix of the symplectic form becomes $\operatorname{diag}\left\{-i I_{q}, i I_{p}\right\}$, with the corresponding direct sum decomposition

$$
S=N_{-} \oplus P_{+} \text {with }\left[N_{-}: P_{+}\right]=0 .
$$

Here $N_{-}$is spanned by the first $q$ vectors of the chosen basis, and $P_{+}$by the last $p$ vectors.

For each vector $u=\left(u_{1}, \ldots, u_{q}, u_{q+1}, \ldots, u_{D}\right) \in S$ let $u_{-}=\left(u_{1}, \ldots, u_{q}\right)$ and $u_{+}=\left(u_{q+1}, \ldots, u_{D}\right)$ be the projections of $u$ on $N_{-}$and $P_{+}$, respectively, (depending on the chosen basis in $S)$. Denote this decomposition $u=\left(u_{-}, u_{+}\right)$, or equally well, $u=u_{-}+u_{+}$as vectors in $S$.

Further, introduce the Hermitian inner products,

$$
\left\langle u_{-}, v_{-}\right\rangle_{-}=u_{1} \bar{v}_{1}+\cdots+u_{q} \bar{v}_{q} \text { and }\left\langle u_{+}, v_{+}\right\rangle_{+}=u_{q+1} \bar{v}_{q+1}+\cdots+u_{D} \bar{v}_{D},
$$

in $N_{-}$and $P_{+}$, respectively, as determined by the vectors

$$
u=\left(u_{-}, u_{+}\right)=u_{-}+u_{+} \text {and } v=\left(v_{-}, v_{+}\right)=v_{-}+v_{+} \text {in } S .
$$

While the subspaces $N_{-}$and $P_{+}$are not unique in $S$, once $N_{-}$is fixed then so is $P_{+}$and also the unitary or Hermitian metrics on both.

Then, for vectors $u, v \in S$,

$$
[u: v]=-i\left\{\left\langle u_{-}, v_{-}\right\rangle_{-}-\left\langle u_{+}, v_{+}\right\rangle_{+}\right\} .
$$

In particular, note that

$$
[u: v]=0 \quad \text { if and only if }\left\langle u_{-}, v_{-}\right\rangle_{-}=\left\langle u_{+}, v_{+}\right\rangle_{+}
$$

and also

$$
[v: v]=0 \quad \text { if and only if }\left\|v_{-}\right\|_{-}=\left\|v_{+}\right\|_{+},
$$

in terms of the norms $\|\cdot\|_{-}$and $\|\cdot\|_{+}$on the Hermitian metric spaces $N_{-}$and $P_{+}$, respectively.

Proof. The formula (2.23) is obvious, since

SO

$$
[u: v]=\left(u_{-}, u_{+}\right)\left(\begin{array}{cc}
-i I_{q} & 0 \\
0 & i I_{p}
\end{array}\right)\left(v_{-}, v_{+}\right)^{*}
$$

as required.

$$
[u: v]=-i\left\{\left\langle u_{-}, v_{-}\right\rangle_{-}-\left\langle u_{+}, v_{+}\right\rangle_{+}\right\}
$$

Lemma 2. Consider a complex symplectic space $S$ with symplectic form [:], and finite dimension $D \geq 1$. Let $\hat{L}$ be a Lagrangian subspace of dimension $\delta \leq \Delta$, where $\Delta=\frac{1}{2}(D-|E x|)$, for invariants $D=p+q, E x=p-q$ as in (2.6).

Then there exists a direct sum decomposition

$$
S=S^{2 \Delta} \oplus S^{|E x|} \text { with }\left[S^{2 \Delta}: S^{|E x|}\right]=0,
$$

with terms having dimension $2 \Delta$ and $|E x|$ and excess of zero and $p-q$, respectively, as in Corollary 1 (2.12), together with a canonical basis $\left\{\hat{e}^{1}, \ldots, \hat{e}^{\Delta}, \hat{e}^{\Delta+1}, \ldots, \hat{e}^{2 \Delta}\right\}$ for the symplectic space $S^{2 \Delta}$, such that

$$
\hat{L}=\operatorname{span}\left\{\hat{e}^{1}, \hat{e}^{2}, \ldots, \hat{e}^{\delta}\right\} \text {. }
$$

Thus $\hat{L}$ lies within the Lagrangian $\Delta$-space $\operatorname{span}\left\{\hat{e}^{1}, \ldots, \hat{e}^{\Delta}\right\} \subset S^{2 \Delta}$. 
If we supplement the canonical basis for $S^{2 \Delta}$ by a diagonalizing basis for $S^{|E x|}$ (as in (2.14)), the symplectic products in $S$ are computed via the $D \times D$ skew-Hermitian matrix (compare (2.10), (2.13) for conventions when $E x \geq 0$ or $E x<0$ )

$$
H=\left(\begin{array}{ccc}
0 & I_{\Delta} & 0 \\
-I_{\Delta} & 0 & 0 \\
0 & 0 & \pm i I_{|E x|}
\end{array}\right) .
$$

Proof. Without loss of generality, we need treat only the case $p \geq q=\Delta$, so $E x=p-q \geq 0$. We choose a basis in $S$ so the skew-Hermitian $D \times D$ matrix of the symplectic form is $\operatorname{diag}\left\{-i I_{q}, i I_{p}\right\}$, just as in Lemma 1 . Then the corresponding direct sum decomposition for $S$ is

$$
S=N_{-} \oplus P_{+} \quad \text { with }\left[N_{-}: P_{+}\right]=0,
$$

and each vector $u \in S$ has the decomposition

$$
u=\left(u_{-}, u_{+}\right) \text {or } u=u_{-}+u_{+} \text {, with } u_{-} \in N_{-}, u_{+} \in P_{+} .
$$

Also introduce the Hermitian inner products $\langle\cdot, \cdot\rangle_{-}$on $N_{-}$and $\langle\cdot, \cdot\rangle_{+}$on $P_{+}$, as in (2.21).

Now take any basis $\left\{e^{1}, \ldots, e^{\delta}\right\}$ for the Lagrangian $\delta$-space $\hat{L} \subset S$ (with $1 \leq \delta \leq$ $\Delta)$, and consider the corresponding decompositions

$$
e^{j}=e_{-}^{j}+e_{+}^{j}, \quad \text { for } j=1, \ldots, \delta .
$$

Then $\hat{L}_{-}=\operatorname{span}\left\{e_{-}^{1}, \ldots, e_{-}^{\delta}\right\} \subset N_{-}$and $\hat{L}_{+}=\operatorname{span}\left\{e_{+}^{1}, \ldots, e_{+}^{\delta}\right\} \subset P_{+}$each have dimension $\delta$, because otherwise there would exist a nonzero vector $w=\left(w_{-}, w_{+}\right) \in$ $\hat{L}$ with either $w_{-}=0$ or $w_{+}=0$. But this is impossible since $w \in \hat{L}$ is a neutral vector, that is, $[w: w]=0$, so $\left\|w_{-}\right\|_{-}=\left\|w_{+}\right\|_{+}$as in (2.25).

Thus both $\left\{e_{-}^{1}, \ldots, e_{-}^{\delta}\right\}$ and $\left\{e_{+}^{1}, \ldots, e_{+}^{\delta}\right\}$ span $\delta$-subspaces in $N_{-}$and $P_{+}$, respectively. Hence, without modifying the notation, we assume that $\left\{e_{-}^{1}, \ldots, e_{-}^{\delta}\right\}$ are orthonormal in $N_{-}$, that is, $\left\langle e_{-}^{j}, e_{-}^{k}\right\rangle_{-}=\delta^{j k}$, and so by (2.24) we also have $\left\langle e_{+}^{j}, e_{+}^{k}\right\rangle_{+}=\delta^{j k}$ for $1 \leq j, k \leq \delta$.

Now suppose $\delta<\Delta$. In this case choose a vector $e^{\delta+1}=\left(e_{-}^{\delta+1}, e_{+}^{\delta+1}\right) \in S$, with $\left\|e_{-}^{\delta+1}\right\|_{-}=\left\|e_{+}^{\delta+1}\right\|_{+}=1$, and further $\left\langle e_{+}^{\delta+1}, e_{+}^{j}\right\rangle_{ \pm}=0$ for all $j=1, \ldots, \delta$. Thus $e^{\delta+1}$ is a non-zero neutral vector, that is, $\left[e^{\delta+1}: e^{\delta+1}\right]=0$, which is linearly independent of $\hat{L}$. But $\left[\hat{L}: e^{\delta+1}\right]=0$, by $(2.24)$, so we can obtain a Lagrangian space defined by $\operatorname{span}\left\{\hat{L}, e^{\delta+1}\right\}$ with a basis $\left\{e^{1}, e^{2}, \ldots, e^{\delta}, e^{\delta+1}\right\}$. We continue this process of augmentation of the basis of $\hat{L}$ until we construct a Lagrangian $\Delta$-space

$$
L=\operatorname{span}\left\{e^{1}, e^{2}, \ldots, e^{\delta}, e^{\delta+1}, \ldots, e^{\Delta}\right\}
$$

with $e^{j}=e_{-}^{j}+e_{+}^{j}$ satisfying

$$
\left\langle e_{-}^{j}, e_{-}^{k}\right\rangle_{-}=\delta^{j k},\left\langle e_{+}^{j}, e_{+}^{k}\right\rangle_{+}=\delta^{j k}, \text { for } 1 \leq j, k \leq \Delta .
$$

Next consider the subspaces $C_{1} \subset S$ and $C_{2} \subset S$ as defined by

$$
C_{1}=\operatorname{span}\left\{e_{-}^{1}, e_{-}^{2}, \ldots, e_{-}^{\Delta}, e_{+}^{1}, e_{+}^{2}, \ldots, e_{+}^{\Delta}\right\},
$$

and within the Hermitian metric space $P_{+}$define

$$
C_{2}=\text { orthocomplement of } \operatorname{span}\left\{e_{+}^{1}, \ldots, e_{+}^{\Delta}\right\} \text { in } P_{+} .
$$


Since $\operatorname{span}\left\{e_{-}^{1}, e_{-}^{2}, \ldots, e_{-}^{\Delta}\right\}=N_{-}$and $\left[\operatorname{span}\left\{e_{+}^{1}, \ldots, e_{+}^{\Delta}\right\}: C_{2}\right]=0$, we see that

$$
S=C_{1} \oplus C_{2} \text { with }\left[C_{1}: C_{2}\right]=0 .
$$

Thus $C_{1}$ and $C_{2}$ are symplectic subspaces of $S$, with dimensions specified by $2 \Delta$ and $p-q$, respectively, and we denote these spaces by

$$
C_{1}=S^{2 \Delta} \text { and } C_{2}=S^{|E x|} .
$$

Since $S^{2 \Delta}$ contains the Lagrangian $\Delta$-space $L$, and $\operatorname{dim} S^{2 \Delta}=2 \Delta$, it follows that $S^{2 \Delta}$ must be isomorphic to the complex symplectic space $\mathbf{C}^{2 \Delta}$ which has excess zero; and hence $S^{|E x|}$ must have excess $p-q$, and, as asserted in the theorem,

$$
S=S^{2 \Delta} \oplus S^{|E x|} \text { with }\left[S^{2 \Delta}: S^{|E x|}\right]=0 .
$$

Finally, we shall define a new basis in $C_{1}=S^{2 \Delta}$ by

$$
\left\{\hat{e}^{1}, \ldots \hat{e}^{\Delta}, \hat{e}^{\Delta+1}, \ldots, \hat{e}^{2 \Delta}\right\}
$$

with

$$
\hat{e}^{j}=\frac{1}{\sqrt{2}}\left(e_{-}^{j}+e_{+}^{j}\right)=\frac{1}{\sqrt{2}} e^{j}
$$

and

$$
\hat{e}^{\Delta+j}=\frac{1}{\sqrt{2} i}\left(e_{-}^{j}-e_{+}^{j}\right), \text { for } j=1, \ldots, \Delta .
$$

Because

$$
e_{-}^{j}=\frac{1}{\sqrt{2}}\left(\hat{e}^{j}+i \hat{e}^{\Delta+j}\right), \quad e_{+}^{j}=\frac{1}{\sqrt{2}}\left(\hat{e}^{j}-i \hat{e}^{\Delta+j}\right),
$$

we see that the $2 \Delta$ vectors $\left\{\hat{e}^{1}, \ldots, \hat{e}^{\Delta}, \hat{e}^{\Delta+1}, \ldots, \hat{e}^{2 \Delta}\right\}$ are linearly independent and $\operatorname{span} S^{2 \Delta}$.

Moreover

$$
\hat{L}=\operatorname{span}\left\{e^{1}, e^{2}, \ldots, e^{\delta}\right\}=\operatorname{span}\left\{\hat{e}^{1}, \hat{e}^{2}, \ldots \hat{e}^{\delta}\right\},
$$

and it is easy to use (2.23), (2.32), (2.35) to compute

$$
\left[\hat{e}^{j}: \hat{e}^{k}\right]=0, \quad\left[\hat{e}^{\Delta+j}: \hat{e}^{\Delta+k}\right]=0
$$

and

$$
\left[\hat{e}^{j}: \hat{e}^{\Delta+k}\right]=\delta^{j k}, \text { for } 1 \leq j, k \leq \Delta .
$$

Hence $\left\{\hat{e}^{1}, \ldots, \hat{e}^{\Delta}, \hat{e}^{\Delta+1}, \ldots, \hat{e}^{2 \Delta}\right\}$ is a canonical basis for $S^{2 \Delta}$, as required.

Corollary 1. Consider a complex sympletic space $S$ with symplectic form [:], and with finite dimension $D \geq 1$. Let $L_{1}$ and $L_{2}$ be two Lagrangian $\delta$-subspaces of $S$ for $1 \leq \delta \leq \Delta$. Then there exists a symplectic automorphism of $S$ which carries $L_{1}$ onto $L_{2}$.

Proof. There exists a decomposition $S=S^{2 \Delta} \oplus S^{|E x|}$, with a corresponding basis $(2.14),(2.27)$, as in Lemma 2 , so there is a canonical basis $\left\{\hat{e}^{1}, \ldots, \hat{e}^{\Delta}, \hat{e}^{\Delta+1}, \ldots, \hat{e}^{2 \Delta}\right\}$ for $S^{2 \Delta}$ with $L_{1}$ given by $\operatorname{span}\left\{\hat{e}^{1}, \ldots, \hat{e}^{\delta}\right\}$. A similar result holds for $L_{2}$; and the symplectic automorphism of $S$, defined by the two specified bases, will carry $L_{1}$ onto $L_{2}$. 
Theorem 2. Let $S$ be a complex symplectic space, with symplectic form [:] and finite dimension $D \geq 1$. Then there exists a complete Lagrangian subspace of $S$ if and only if $S$ has excess $E x=0$.

In case $E x=0$ then $\operatorname{dim} S=D=2 \Delta$ is even, and furthermore a Lagrangian subspace $L$ is then complete if and only if $\operatorname{dim} L=\Delta$.

Proof. Assume $E x=0$ so that $S$ is the complexification of $\mathbf{R}^{2 \Delta}$. Then there exists a canonical basis ( $\operatorname{see}(2.7))\left\{e^{1}, \ldots, e^{\Delta}, e^{\Delta+1}, \ldots, e^{2 \Delta}\right\}$ for $S$ such that the corresponding skew-Hermitian matrix of the symplectic products is

$$
\left(\begin{array}{cc}
0 & I_{\Delta} \\
-I_{\Delta} & 0
\end{array}\right)
$$

In this case

$$
L=\operatorname{span}\left\{e^{1}, \ldots, e^{\Delta}\right\}
$$

is a complete Lagrangian subspace of $S$. For suppose $u=c_{1} e^{1}+\cdots+c_{\Delta} e^{\Delta}+$ $c_{\Delta+1} e^{\Delta+1}+\cdots+c_{2 \Delta} e^{2 \Delta}$ satisfies $[u: L]=0$. Then because of the canonical duality $\left[e^{j}: e^{\Delta+k}\right]=\delta^{j k}$ for $1 \leq j, k \leq \Delta$, we conclude that $c_{\Delta+1}=c_{\Delta+2}=\cdots=c_{2 \Delta}=0$ and so $u \in L$.

Also in case $E x=0$ we know, from Lemma 2 above, that for each Lagrangian $\hat{L}$ of dimension $\delta \leq \Delta$ there exists a canonical basis $\left\{\hat{e}^{1}, \ldots, \hat{e}^{\Delta}, \hat{e}^{\Delta+1}, \ldots, \hat{e}^{2 \Delta}\right\}$ such that $\hat{L}=\operatorname{span}\left\{\hat{e}^{1}, \ldots, \hat{e}^{\delta}\right\}$. If $\delta=\Delta$ then, as in the previous argument, $\hat{L}$ is complete. If $\delta<\Delta$, then $\hat{L}$ is not complete, since $\left[\hat{e}^{\delta+1}: \hat{L}\right]=0$ yet $\hat{e}^{\delta+1} \notin \hat{L}$. (Clearly $\operatorname{dim} \hat{L}$ cannot exceed $\Delta$, according to (2.6).) Hence $\hat{L}$ is complete if and only if $\operatorname{dim} \hat{L}=\Delta$.

Finally, consider the case where $S$ has $E x \neq 0$, say $E x=p-q>0$. Then $\Delta=\min \{p, q\}=q$. Again by Lemma 2 , for each Lagrangian subspace $\hat{L} \subset S$ there exists a basis of $S$ (see $(2.14)),\left\{\hat{e}^{1}, \ldots, \hat{e}^{2 \Delta}, \epsilon^{1}, \ldots, \epsilon^{E x}\right\}$ such that the corresponding skew-Hermitian matrix is

$$
\left(\begin{array}{ccc}
0 & I_{\Delta} & 0 \\
-I_{\Delta} & 0 & 0 \\
0 & 0 & i I_{E x}
\end{array}\right) .
$$

Moreover, $\hat{L} \subset \operatorname{span}\left\{\hat{e}^{1}, \ldots, \hat{e}^{\Delta}\right\}$. But then the vector $\epsilon^{1}$ satisfies $\left[\epsilon^{1}: \hat{L}\right]=0$, and yet $\epsilon^{1} \notin \hat{L}$. Therefore $\hat{L}$ is not a complete Lagrangian subspace of $S$, and the theorem is proved.

The final three theorems of this section all deal with complex symplectic spaces $S$ having finite dimension $D \geq 1$ and excess $E x=0$. These results are directly applicable to the study of self-adjoint boundary value problems, although the presentation here will be entirely algebraic.

The next Theorem 3 relates the investigations of von Neumann [AG], [DS], [NA] on isometries of unitary spaces with our treatment of complete Lagrangian subspaces of a complex symplectic space. The notation and special formulations follow the developments in Lemma 1 of Theorem 2.

Theorem 3. Let $S$ be a complex symplectic space, with symplectic form [:], having finite dimension $D \geq 1$ and excess $E x=0$.

Fix a symplectic orthogonal decomposition

$$
S=N_{-} \oplus P_{+} \text {, with }\left[N_{-}: P_{+}\right]=0,
$$


such that

$$
\left\langle u_{-}, v_{-}\right\rangle_{-}=i\left[u_{-}: v_{-}\right] \text {on } N_{-}, \text {and }\left\langle u_{+}, v_{+}\right\rangle_{+}=-i\left[u_{+}: v_{+}\right] \text {on } P_{+}
$$

are Hermitian inner products on the subspaces $N_{-}$and $P_{+}$, respectively. Because $E x=0 D=2 \Delta$, then $\operatorname{dim} N_{-}=\operatorname{dim} P_{+}=\Delta$ and such decompositions of $S$ exist, see Lemma 1 of Theorem 2 above.

Then the following formula holds for all $u, v \in S$ :

$$
[u: v]=-i\left\langle u_{-}, v_{-}\right\rangle_{-}+i\left\langle u_{+}, v_{+}\right\rangle_{+} \cdot
$$

Here $u=u_{-}+u_{+}$(also denoted by the ordered pair $u=\left(u_{-}, u_{+}\right)$) is the unique decomposition of $u \in S$ with $u_{-} \in N_{-}, u_{+} \in P_{+}$, and similarly for $v \in S$.

In this situation there exists a natural bijection between the set $\{U\}$ of all unitary isometries of $N_{-}$onto $P_{+}$as Hermitian metric spaces, and the set $\{L\}$ of all complete Lagrangian subspaces of $S$. Namely, for each unitary surjection

$$
U: N_{-} \rightarrow P_{+},
$$

the corresponding complete Lagrangian subspace of $S$ is

$$
L=\operatorname{graph} U
$$

that is, $u=u_{-}+u_{+} \in L$ if and only if $u_{+}=U u_{-}$, which can also be written

$$
u=\left(u_{-}, u_{+}\right)=\left(u_{-}, U u_{-}\right) \in \operatorname{graph} U .
$$

Proof. The formula (2.40) follows easily, since for $u, v \in S$ $[u: v]=\left[u_{-}+u_{+}: v_{-}+v_{+}\right]=\left[u_{-}: v_{-}\right]+\left[u_{+}: v_{+}\right]=-i\left\langle u_{-}, v_{-}\right\rangle_{-}+i\left\langle u_{+}, v_{+}\right\rangle_{+}$, and consequently we have the useful results (see (2.24), (2.25))

$$
[u: v]=0 \text { if and only if }\left\langle u_{-}, v_{-}\right\rangle_{-}=\left\langle u_{+}, v_{+}\right\rangle_{+} .
$$

Let $U: N_{-} \rightarrow P_{+}$be a unitary map of $N_{-}$onto $P_{+}$, as Hermitian metric spaces of dimension $\Delta$. Now define the set $L \subset S$ by

$$
L=\left\{u=u_{-}+u_{+} \in S \mid u_{+}=U u_{-}\right\}=\operatorname{graph} U .
$$

Clearly $L$ is a linear subspace of $S$, since $U$ is linear. Then for vectors $u, v \in L$ we compute

$$
[u: v]=i\left\{\left\langle u_{+}, v_{+}\right\rangle_{+}-\left\langle u_{-}, v_{-}\right\rangle_{-}\right\}=0,
$$

since $\left\langle u_{+}, v_{+}\right\rangle_{+}=\left\langle U u_{-}, U v_{-}\right\rangle_{+}=\left\langle u_{-}, v_{-}\right\rangle_{-}$. Hence $L$ is a Lagrangian subspace of the complex symplectic space $S$, and we next verify that $L$ is complete.

Let $\left\{e_{-}^{1}, \ldots, e_{-}^{\Delta}\right\}$ be an orthonormal basis of the Hermitian metric space $N_{-}$, so then $\left\{e_{+}^{1}, \ldots, e_{+}^{\Delta}\right\}$ is an orthonormal basis for $P_{+}$, where we define $e_{+}^{j}=U e_{-}^{j}$ for $j=1, \ldots, \Delta$. Suppose $f=f_{-}+f_{+} \in S$ satisfies $[f: L]=0$. Then each $e^{j}=e_{-}^{j}+e_{+}^{j} \in L$, so

$$
\begin{aligned}
{\left[f: e_{-}^{j}+e_{+}^{j}\right] } & =\left[f_{-}: e_{-}^{j}\right]+\left[f_{+}: e_{+}^{j}\right] \\
& =-i\left\langle f_{-}, e_{-}^{j}\right\rangle_{-}+i\left\langle f_{+}, e_{+}^{j}\right\rangle_{+}=0 .
\end{aligned}
$$

Thus

$$
\left\langle f_{-}, e_{-}^{j}\right\rangle_{-}=\left\langle f_{+}, e_{+}^{j}\right\rangle_{+} \text {for } j=1, \ldots, \Delta .
$$

But

$$
\left\langle f_{-}, e_{-}^{j}\right\rangle_{-}=\left\langle U f_{-}, e_{+}^{j}\right\rangle_{+}
$$


so we conclude that $f_{+}=U f_{-}$. Hence $f \in L$, and so $L$ is a complete Lagrangian space.

On the other hand let $L$ now be a given complete Lagrangian subspace of $S$, so $\operatorname{dim} L=\Delta$. We seek to construct an appropriate unitary isometry $U$ of $N_{-}$onto $P_{+}$, as Hermitian metric spaces.

Take a basis $\left\{e^{1}, \ldots, e^{\Delta}\right\}$ for $L$ and write $e^{j}=e_{-}^{j}+e_{+}^{j}$ for $j=1, \ldots, \Delta$, as usual. Then $\left\{e_{-}^{1}, \ldots, e_{-}^{\Delta}\right\}$ must be independent and so a basis for $N_{-}$. Because suppose these vectors were dependent in $N_{-}$and some non-trivial linear combination were zero, say $w_{-}=c_{1} e_{-}^{1}+\cdots+c_{\Delta} e_{-}^{\Delta}=0$ for complex constants $c_{1}, \ldots, c_{\Delta}$ not all zero. Then with these same constants define $w=c_{1} e^{1}+\cdots+c_{\Delta} e^{\Delta} \in L$; so $[w: w]=0$ and $\left\|w_{-}\right\|_{-}=\left\|w_{+}\right\|_{+}=0$ (see (2.43)). This calculation $w=w_{-}+w_{+}=0$ contradicts the independence of the basis $\left\{e^{1}, \ldots, e^{\Delta}\right\}$ of $L$, so we conclude that $\left\{e_{-}^{1}, \ldots, e_{-}^{\Delta}\right\}$ is a basis for $N_{-}$, and similarly $\left\{e_{+}^{1}, \ldots, e_{+}^{\Delta}\right\}$ is a basis for $P_{+}$.

In fact, upon re-selecting a suitable linear combination of the vectors $e^{1}, \ldots, e^{\Delta}$ we obtain a new basis for $L$ (without changing notation) so that we can assume $\left\{e^{1}, \ldots, e^{\Delta}\right\}$ has the property that $\left\{e_{-}^{1}, \ldots, e_{-}^{\Delta}\right\}$ is an orthonormal basis for the Hermitian metric space $N_{-}$. However, $\left\langle e_{-}^{j}, e_{-}^{k}\right\rangle_{-}=\left\langle e_{+}^{j}, e_{+}^{k}\right\rangle_{+}=\delta^{j k}$ for $1 \leq j, k \leq$ $\Delta$ (see $(2.43)$ ), and hence $\left\{e_{+}^{1}, \ldots, e_{+}^{\Delta}\right\}$ is an orthonormal basis for the Hermitian metric space $P_{+}$.

Now define the required unitary surjection $U: N_{-} \rightarrow P_{+}$by

$$
U: e_{-}^{j} \rightarrow U e_{-}^{j}=e_{+}^{j} \text { for } 1, \ldots, \Delta .
$$

We claim that $L=$ graph $U$. Indeed, let $u=u_{-}+u_{+} \in L$, that is, $[u: L]=0$, so then $\left\langle u_{-}, e_{-}^{j}\right\rangle_{-}=\left\langle u_{+}, e_{+}^{j}\right\rangle_{+}$. But since $U$ is unitary $\left\langle u_{-}, e_{-}^{j}\right\rangle_{-}=\left\langle U u_{-}, U e_{-}^{j}\right\rangle_{+}=$ $\left\langle U u_{-}, e_{+}^{j}\right\rangle_{+}$. This proves that $U u_{-}=u_{+}$, so $u \in \operatorname{graph} U$, and thus $L \subset$ graph $U$. But $L$ and graph $U$ are each $\Delta$-dimensional subspaces of $S$, and therefore $L=$ graph $U$.

Finally, the surjective map $\{U\} \rightarrow\{L\}$ defined by $L=$ graph $U$ is injective, because two different unitary maps of $N_{-}$into $P_{+}$must have different graphs.

The next Theorem 4, called the Balanced intersection principle, is one of the major results of our investigation; and it imposes restrictions on the kinds of boundary conditions that can be relevant in the applications to self-adjoint boundary value problems $[\mathrm{EI}],[\mathrm{EM}]$. However, since this result is purely algebraic, we present it here without references to the function spaces $D\left(T_{\max }\right), D\left(T_{\min }\right)$, and $\mathbf{S}=D\left(T_{\max }\right) / D\left(T_{\min }\right)$ as introduced in Example 3 of Section 1, see (1.27), (1.31) above, or more elaborately in the Appendix.

Theorem 4 (Balanced intersection principle). Let $S$ be a complex symplectic space with symplectic form [:], having a finite dimension $D \geq 1$, and a prescribed direct sum decomposition (as in (2.11)),

$$
S=S_{-} \oplus S_{+} \text {with }\left[S_{-}: S_{+}\right]=0 .
$$

Assume that the symplectic invariants of $S$ satisfy

$$
D=2 \Delta, \quad E x=0,
$$

and denote the corresponding invariants of $S_{ \pm}$by $\Delta_{ \pm}$and Ex $x_{ \pm}$, respectively (see (2.16) and (2.17)). 
Then, for each complete Lagrangian space $L$ in $S$, the balanced intersection principle holds:

$$
0 \leq \Delta_{-}-\operatorname{dim} L \cap S_{-}=\Delta_{+}-\operatorname{dim} L \cap S_{+} \leq \min \left\{\Delta_{-}, \Delta_{+}\right\} .
$$

Proof. Without loss of generality we shall assume that $\Delta_{+} \geq \Delta_{-} \geq 0$, so

$$
\min \left\{\Delta_{-}, \Delta_{+}\right\}=\Delta_{-} .
$$

Also it is clear that $E x_{+}=-E x_{-}$, since $E x=0$ for $S$.

First we present some preliminary observations and calculations, leading to the inequalities (2.50) below, in order to simplify the main arguments of the proof of this theorem. We also emphasize that a complete Lagrangian $L$ in $S$ must have $\operatorname{dim} L=\Delta$, and, conversely, each Lagrangian $\Delta$-space is a complete Lagrangian.

Since $L \cap S_{-}$is a Lagrangian subspace of the symplectic space $S_{-}$, it follows that $0 \leq \operatorname{dim} L \cap S_{-} \leq \Delta_{-}$, and a similar result holds for $L \cap S_{+}$in the symplectic space $S_{+}$, namely, $0 \leq \operatorname{dim} L \cap S_{+} \leq \Delta_{+}$. Further we note that there are $\Delta=$ $\Delta_{-}+\Delta_{+}+\left|E x_{+}\right|$independent vectors in $L$ (see (2.19)), and we shall next proceed to select bases in $S_{-}$and in $S_{+}$, adapted to the Lagrangian subspaces $L \cap S_{-}$and $L \cap S_{+}$, respectively.

To accomplish this construction, take a basis for $S_{-}$, as in (2.14),

$$
\left\{e_{-}^{1}, e_{-}^{2}, \ldots, e_{-}^{\Delta_{-}} e_{-}^{\Delta_{-}+1}, \ldots, e_{-}^{2 \Delta_{-}}, \epsilon_{-}^{1}, \epsilon_{-}^{2}, \ldots, \epsilon_{-}^{\left|E x_{-}\right|}\right\}
$$

where the first $2 \Delta_{-}$vectors constitute a canonical basis for a classical $\left(2 \Delta_{-}\right)$subspace of $S_{-}$, and the last $\left|E x_{-}\right|$vectors span a subspace of $S_{-}$that contains no non-zero neutral vectors (see Corollary 1 of Theorem 1, and Lemma 2 of Theorem 2 above). In fact, we require that the initial sequence, consisting of $\left|\operatorname{dim} L \cap S_{-}\right|$ vectors from $\left\{e_{-}^{1}, \ldots, e_{-}^{\Delta_{-}}\right\}$, is a basis for $L \cap S_{-}$. A similar basis is also constructed for $S_{+}$, similarly adapted to $L \cap S_{+}$.

In terms of these bases for $S_{-}$and $S_{+}$we can expand each of the $\Delta$ vectors $f^{1}$, $f^{2}, \ldots, f^{\Delta}$ of a basis for $L$, namely

$$
\begin{aligned}
f^{s}= & \alpha_{1}^{s} e_{-}^{1}+\alpha_{2}^{s} e_{-}^{2}+\cdots+\alpha_{\Delta_{-}}^{s} e_{-}^{\Delta_{-}}+\alpha_{\Delta_{-}+1}^{s} e_{-}^{\Delta_{-}+1}+\cdots+\alpha_{2 \Delta_{-}}^{s} e_{-}^{2 \Delta_{-}} \\
& +\gamma_{1}^{s} \epsilon_{-}^{1}+\cdots+\gamma_{\left|E x_{-}\right|}^{s} \epsilon_{-}^{\left|E x_{-}\right|} \\
& + \text {similar terms from } S_{+}, \quad \text { for } s=1,2, \ldots, \Delta .
\end{aligned}
$$

We denote by $f^{s}\left(\bmod S_{+}\right)$the projection of the vector $f^{s} \in S$ into $S_{-}$(merely delete all the terms from $S_{+}$in the expansion $(2.47)$ ), for $s=1,2, \ldots, \Delta$. Now observe that all $f^{s}\left(\bmod S_{+}\right)$span a subspace of $S_{-}$which is just the projection of $L$ into $S_{-}$, and we denote this by $L\left(\bmod S_{+}\right)$. Clearly $L\left(\bmod S_{+}\right)$is a linear subspace with dimension $\leq \operatorname{dim} S_{-}=2 \Delta_{-}+\left|E x_{-}\right|$.

By elementary linear algebra, $L$ must contain (at least) $\Delta-\left(2 \Delta_{-}+\left|E x_{-}\right|\right)=$ $\Delta_{+}-\Delta_{-}$independent vectors that lie within $S_{+}$. Since this type of argument will be used several times in this proof, we present the calculations in full detail this first time. That is, consider the matrix of complex numbers

$$
\left(\begin{array}{ccccccc}
\alpha_{1}^{1} & \alpha_{2}^{1} & \ldots & \alpha_{2 \Delta_{-}}^{1} & \gamma_{1}^{1} & \ldots & \gamma_{\left|E x_{-}\right|}^{1} \\
\vdots & & & & & & \\
\alpha_{1}^{\Delta} & \alpha_{2}^{\Delta} & \ldots & \alpha_{2 \Delta_{-}}^{\Delta} & \gamma_{1}^{\Delta} & \ldots & \gamma_{\left|E x_{-}\right|}^{\Delta}
\end{array}\right),
$$


with $\Delta$ rows, but with rank $\leq \operatorname{dim} L\left(\bmod S_{+}\right)$. Thus the rank of this matrix is $\leq \operatorname{dim} S_{-}$, and

$$
\operatorname{dim} S_{-} \leq 2 \Delta_{-}+\left|E x_{-}\right| \leq \Delta_{-}+\Delta_{+}+\left|E x_{-}\right|=\Delta .
$$

Hence elementary (nonsingular) row transformations in (2.48) lead to a matrix with (at least) the first $\Delta-\left(2 \Delta_{-}+\left|E x_{-}\right|\right)=\Delta_{+}-\Delta_{-}$rows consisting of zeros. The same elementary row transformations applied to the full matrix of all coefficients of $\left\{f^{s}\right\}$, as in (2.47), then yield (at least) $\Delta_{+}-\Delta_{-}$independent vectors in $L$ which lie within $S_{+}$, since they have zero projections in $S_{-}$.

Thus we have established that

$$
\operatorname{dim} L \cap S_{+} \geq \Delta_{+}-\Delta_{-} \geq 0,
$$

and hence the preliminary inequalities (both terms within $\left[0, \min \left\{\Delta_{-}, \Delta_{+}\right\}\right]$)

$$
0 \leq \Delta_{-}-\operatorname{dim} L \cap S_{-}, \Delta_{+}-\operatorname{dim} L \cap S_{+} \leq \min \left\{\Delta_{-}, \Delta_{+}\right\}
$$

hold.

As a final preliminary, before starting the main inductive argument of the proof, we resolve the extreme case $\Delta_{-}=0$. Here $\operatorname{dim} L \cap S_{-}=\Delta_{-}=0$. Also, by (2.50),

$$
\Delta_{+}-\operatorname{dim} L \cap S_{+} \leq \Delta_{-}=0, \quad \text { so } \operatorname{dim} L \cap S_{+}=\Delta_{+} .
$$

Therefore, in the case $\Delta_{-}=0$, we have shown that

$$
0=\Delta_{-}-\operatorname{dim} L \cap S_{-}=\Delta_{+}-\operatorname{dim} L \cap S_{+}=\Delta_{-},
$$

and so the conclusion of Theorem 4 holds. Of course, the same result holds if $\Delta_{+}=0$, since $\Delta_{+} \geq \Delta_{-} \geq 0$ by our standing hypothesis.

We now return to the main line of argument of the proof of Theorem 4 , but we re-state the desired conclusion in the format, for a Lagrangian $\Delta$-space $L \subset S$,

$$
\operatorname{dim} L \cap S_{-}=\Delta_{-}-\ell \text { if and only if } \operatorname{dim} L \cap S_{+}=\Delta_{+}-\ell
$$

for each integer $\ell=0,1,2, \ldots, \Delta_{-}=\min \left\{\Delta_{-}, \Delta_{+}\right\}$. Since we have already demonstrated this conclusion for the case $\Delta_{-}=0$, we henceforth shall assume that $\Delta_{+} \geq \Delta_{-} \geq 1$. In our analysis we shall first prove the required conclusion (2.51) for $\ell=0$, then for $\ell=1$, etc., up to $\ell=\Delta_{-}-1$. The final step $\ell=\Delta_{-}$will be analysed at the end of the proof.

Main argument for the proof of Theorem 4. In the first step consider the case $\ell=0$ (which is relevant since $\ell=0 \leq \Delta_{-}-1$ when $\Delta_{-} \geq 1$ ). Let $\operatorname{dim} L \cap S_{-}=\Delta_{-}$and show that $\operatorname{dim} L \cap S_{+}=\Delta_{+}$, and vice versa.

As before in (2.46), take a basis for $S_{-}$

$$
\left\{e_{-}^{1}, \ldots, e_{-}^{\Delta_{-}} e_{-}^{\Delta_{-}+1}, \ldots, e_{-}^{2 \Delta_{-}}, \epsilon_{-}^{1}, \ldots, \epsilon_{-}^{\left|E x_{-}\right|}\right\}
$$

and similarly a basis for $S_{+}$. Also require that the Lagrangian $\Delta$-space $L$ meets $S_{-}$ in

$$
L \cap S_{-}=\operatorname{span}\left\{e_{-}^{1}, \ldots, e_{-}^{\Delta_{-}}\right\} .
$$

Now select a basis for $L$ in the format

$$
L=\operatorname{span}\left\{e_{-}^{1}, \ldots, e_{-}^{\Delta_{-}}, h^{s}\right\}, \quad \text { with } s=1,2, \ldots, \Delta_{+}+\left|E x_{-}\right| .
$$

Because $\left[e_{-}^{j}: e_{-}^{\Delta_{-}+k}\right]=\delta^{j k}$ for $1 \leq j, k \leq \Delta_{-}$, we can demand that $h^{s}\left(\bmod S_{+}\right)$ do not involve $\left\{e_{-}^{1}, \ldots, e_{-}^{\Delta_{-}}\right\}$or $\left\{e_{-}^{\Delta_{-}+1}, \ldots, e_{-}^{2 \Delta_{-}}\right\}$, and hence depend only on $\epsilon_{-}^{1}, \ldots, \epsilon_{-}^{\left|E x_{-}\right|}$. In such a case $\left\{h^{s}\right\}$ constitute a set of $\Delta_{+}+\left|E x_{-}\right|$independent 
vectors in $L$, yet these project to $h^{s}\left(\bmod S_{+}\right)$, whose span in $S_{-}$has a dimension $\leq\left|E x_{-}\right|$. Therefore by elementary methods of linear algebra, as described earlier, there are (at least) $\left(\Delta_{+}+\left|E x_{-}\right|\right)-\left|E x_{-}\right|=\Delta_{+}$independent vectors in $L \cap S_{+}$. Hence $\operatorname{dim} L \cap S_{+} \geq \Delta_{+}$, and thus necessarily $\operatorname{dim} L \cap S_{+}=\Delta_{+}$.

However, there is an entirely similar proof of the converse:

$$
\text { If } \operatorname{dim} L \cap S_{+}=\Delta_{+} \text {, then } \operatorname{dim} L \cap S_{-}=\Delta_{-} .
$$

(This is not prejudiced by our assumption that $\Delta_{+} \geq \Delta_{-} \geq 1$, and we omit the details.) Therefore we can now assert that

$$
\operatorname{dim} L \cap S_{-}=\Delta_{-} \text {if and only if } \operatorname{dim} L \cap S_{+}=\Delta_{+},
$$

and the conclusion (2.51) of Theorem 4 holds for $\ell=0$, regardless of the value of $\Delta_{-} \geq 1$.

In the next step consider the case $\ell=1$ (and here we can demand that $\Delta_{-} \geq 2$, since we are here assuming that $\ell \leq \Delta_{-}-1$ ). Following the methods of the preceding step (but now with $\Delta_{-} \geq 2$ ), we shall show that

$$
\operatorname{dim} L \cap S_{-}=\Delta_{-}-1 \text { implies that } \operatorname{dim} L \cap S_{+}=\Delta_{+}-1
$$

(and vice versa).

Assume $\operatorname{dim} L \cap S_{-}=\Delta_{-}-1$ and take a basis for $L$, so that

$$
L=\operatorname{span}\left\{e_{-}^{1}, \ldots, e_{-}^{\Delta_{-}-1}, h^{s}\right\},
$$

for $s=1,2, \ldots, \Delta_{+}+1+\left|E x_{-}\right|$, so

$$
\operatorname{dim} L=\Delta=\left(\Delta_{-}-1\right)+\left(\Delta_{+}+1+\left|E x_{-}\right|\right) .
$$

Again we can require that each $h^{s}$ depends only on $\epsilon_{-}^{1}, \ldots, \epsilon_{-}^{\left|E x_{-}\right|}$, and possibly $e_{-}^{\Delta_{-}}$and its canonical dual or conjugate $e_{-}^{2 \Delta_{-}}$. Hence $h^{s}$ projects to $h^{s}\left(\bmod S_{+}\right)$, whose span in $S_{-}$has dimension $\leq\left|E x_{-}\right|+2$. Then, as in the previous step, we conclude that

$$
\operatorname{dim} L \cap S_{+} \geq\left(\Delta_{+}+1+\left|E x_{-}\right|\right)-\left(\left|E x_{-}\right|+2\right)=\Delta_{+}-1 .
$$

Hence $\operatorname{dim} L \cap S_{+}$is either $\left(\Delta_{+}-1\right)$ or $\Delta_{+}$. But if $\operatorname{dim} L \cap S_{+}=\Delta_{+}$, then the previous step asserts that $\operatorname{dim} L \cap S_{-}=\Delta_{-}$, which contradicts the hypothesis that $\operatorname{dim} L \cap S_{-}=\Delta_{-}-1$ in this step. Therefore we see that

$$
\operatorname{dim} L \cap S_{-}=\Delta_{-}-1 \text { implies that } \operatorname{dim} L \cap S_{+}=\Delta_{+}-1 .
$$

The converse is demonstrated similarly, and so the theorem is proved for the case $\ell=1$, for all $\Delta_{-} \geq 2$.

For the general case $\Delta_{-} \geq 3$ we shall prove that (2.51) holds for all $\ell=$ $0,1,2, \ldots, \Delta_{-}-1$ by means of an induction argument on $\ell$. Certainly we have already verified (2.51) for $\ell=0$ and $\ell=1$.

Now we assume the induction hypothesis:

$$
\begin{array}{r}
\operatorname{dim} L \cap S_{-}=\Delta_{-}-\ell \quad \text { if and only if } \operatorname{dim} L \cap S_{+}=\Delta_{+}-\ell \\
\left.\quad \text { for } \ell=0,1, \ldots, k \text { (any positive integer } k \leq \Delta_{-}-2\right),
\end{array}
$$

and we shall prove that the above assertion is true for $\ell=k+1$. 
For this purpose assume that $\operatorname{dim} L \cap S_{-}=\Delta_{-}-(k+1)$, and we shall prove that $\operatorname{dim} L \cap S_{+}=\Delta_{+}-(k+1)$, and also the converse. We take the bases in $S_{-}$ and $S_{+}$, as in (2.46), adapted to the Lagrangian $\Delta$-space $L$, so

$$
L \cap S_{-}=\operatorname{span}\left\{e_{-}^{1}, e_{-}^{2}, \ldots, e_{-}^{\Delta_{-}-(k+1)}\right\}
$$

and

$$
\begin{aligned}
& L=\operatorname{span}\left\{e_{-}^{1}, e_{-}^{2}, \ldots, e_{-}^{\Delta_{-}(k+1)}, h^{s}\right\} \\
& \text { for } s=1,2, \ldots, \Delta_{+}+(k+1)+\left|E x_{-}\right|
\end{aligned}
$$

so

$$
\operatorname{dim} L=\left[\Delta_{-}-(k+1)\right]+\left[\Delta_{+}+(k+1)+\left|E x_{-}\right|\right]=\Delta .
$$

The vectors $h^{s}$ have expansions, in the basis of $S=S_{-} \oplus S_{+}$, which do not involve $e_{-}^{1}, e_{-}^{2}, \ldots, e_{-}^{\Delta_{-}(k+1)}$ or their dual vectors $e_{-}^{\Delta_{-}+1}, \ldots, e_{-}^{2 \Delta_{-}(k+1)}$ in $S_{-}$. Thus each $h^{s}\left(\bmod S_{+}\right)$depends on only $2(k+1)+\left|E x_{-}\right|$basis vectors in $S_{-}$, namely on $e_{-}^{\Delta_{-}-k}, \ldots, e_{-}^{\Delta_{-}}$and their duals $e_{-}^{2 \Delta_{-}-k}, \ldots, e^{2 \Delta_{-}}$, as well as the vectors $\epsilon_{-}^{1}, \ldots, \epsilon_{-}^{\left|E x_{-}\right|}$. Therefore

$$
\operatorname{dim} \operatorname{span}\left\{h^{s}\right\}\left(\bmod S_{+}\right) \leq 2(k+1)+\left|E x_{-}\right| .
$$

Just as in earlier cases we note that upon a re-selection of the vectors $h^{s}$ in the basis for $L$, we get

$$
\operatorname{dim} L \cap S_{+} \geq\left[\Delta_{+}+(k+1)+\left|E x_{-}\right|\right]-\left[2(k+1)+\left|E x_{-}\right|\right],
$$

so

$$
\operatorname{dim} L \cap S_{+} \geq \Delta_{+}-(k+1) \text {. }
$$

But the alternatives of $\operatorname{dim} L \cap S_{+} \geq \Delta_{+}-k$ are ruled out because, by the induction hypothesis, they lead to the conclusion $\operatorname{dim} L \cap S_{-} \geq \Delta_{-}-k$, which is a contradiction. Therefore we conclude that

$$
\operatorname{dim} L \cap S_{+}=\Delta_{+}-(k+1),
$$

as required. The converse argument is similar upon interchanging the roles of $S_{-}$ and $S_{+}$.

Through this induction proof we have demonstrated the validity of (2.51) for all $\Delta_{-} \geq 1$ and all $\ell=0,1, \ldots, \Delta_{-}-1$.

Finally, consider the last step $\ell=\Delta_{-}$(with $\Delta_{-} \geq 1$ ), which we display in some detail since the proof of this case is slightly different from the previous cases. Here we assume that $\operatorname{dim} L \cap S_{-}=\Delta_{-}-\ell=\Delta_{-}-\Delta_{-}=0$, and seek to prove that $\operatorname{dim} L \cap S_{+}=\Delta_{+}-\Delta_{-} \geq 0$.

Again take a basis $\left\{f^{s}\right\}$ for the Lagrangian $\Delta$-space $L$, with $s=1,2, \ldots, \Delta=$ $\Delta_{+}+\Delta_{-}+\left|E x_{-}\right|$. Here $f^{s}$ projects to $f^{s}\left(\bmod S_{+}\right)$, which lies within the symplectic space $S_{-}$of dimension $2 \Delta_{-}+\left|E x_{-}\right|$. Hence $\operatorname{dim} L \cap S_{+} \geq \Delta-\left(2 \Delta_{-}+\right.$ $\left.\left|E x_{-}\right|\right)=\Delta_{+}-\Delta_{-}$. But, as proved above, if $\operatorname{dim} L \cap S_{+}=\Delta_{+}-\Delta_{-}+1$, then $\operatorname{dim} L \cap S_{-}=\Delta_{-}-\left(\Delta_{-}-1\right)=1$, which contradicts the assumption in this case that $\operatorname{dim} L \cap S_{-}=0$. Continuing to retrace the other alternatives of

$$
\operatorname{dim} L \cap S_{+}=\Delta_{+}-\left(\Delta_{-}-2\right), \Delta_{+}-\left(\Delta_{-}-3\right), \ldots, \Delta_{+}-1, \Delta_{+},
$$

we eliminate all these possibilities because they contradict the results already obtained in the cases $\ell=\Delta_{-}-2, \Delta_{-}-3, \ldots, 2,1,0$. Therefore we conclude that $\operatorname{dim} L \cap S_{+}=\Delta_{+}-\Delta_{-}$, as required. 
The converse assertion is particularly simple in this case $\ell=\Delta_{-}$. Assume $\operatorname{dim} L \cap S_{+}=\Delta_{+}-\Delta_{-}$. Certainly $\operatorname{dim} L \cap S_{-} \geq \Delta_{-}-\Delta_{-} \geq 0$. But the alternatives, consisting of

$$
\operatorname{dim} L \cap S_{-}=1,2, \ldots, \Delta_{-},
$$

give rise to the conclusions of the prior steps

$$
\ell=\Delta_{-}-1, \Delta_{-}-2, \ldots, 2,1,0,
$$

and each of these possibilities contradicts the assumption that $\operatorname{dim} L \cap S_{+}=\Delta_{+}-$ $\Delta_{-}$. Thus we conclude that $\operatorname{dim} L \cap S_{-}=0$, as required.

Therefore we have proved the theorem:

$$
\operatorname{dim} L \cap S_{-}=\Delta_{-}-\ell \text { if and only if } \operatorname{dim} L \cap S_{+}=\Delta_{+}-\ell,
$$

for each integer $\ell=0,1, \ldots, \min \left\{\Delta_{-}, \Delta_{+}\right\}$.

Definition 3. Consider the complex symplectic space $S$ with finite dimension $D \geq$ 1 , and a prescribed direct sum decomposition

$$
S=S_{-} \oplus S_{+} \quad \text { with }\left[S_{-}: S_{+}\right]=0,
$$

and assume $D=2 \Delta$ and the excess $E x=0$ (so $E x_{-}=-E x_{+}$), as in the notation of Theorem 4.

For each complete Lagrangian subspace $L \subset S$ (so $\operatorname{dim} L=\Delta$ ) define the coupling grade of $L$,

$$
\text { grade } L=\Delta_{-}-\operatorname{dim} L \cap S_{-}=\Delta_{+}-\operatorname{dim} L \cap S_{+} .
$$

Also define the necessary coupling of $L$,

(2.66) Nec-coupling $L=\Delta-\operatorname{dim} L \cap S_{-}-\operatorname{dim} L \cap S_{+}=2 \operatorname{grade} L+\left|E x_{ \pm}\right|$;

note that $\left|E x_{-}\right|=\left|E x_{+}\right|=\left|E x_{ \pm}\right|$since $E x=0$; and $\Delta=\Delta_{+}+\Delta_{-}+\left|E x_{ \pm}\right|$.

It is clear that in the terminology of Definition 3,

$$
0 \leq \text { grade } L \leq \min \left\{\Delta_{-}, \Delta_{+}\right\} \text {and }\left|E x_{ \pm}\right| \leq \text {Nec-coupling } L \leq \Delta .
$$

In order to emphasize these extreme cases, and in anticipation of the terminology later adopted for boundary conditions at the left and right endpoint spaces $\left(S_{-}\right.$ and $S_{+}$, respectively), we present the next definition.

Definition 4. Consider the complex symplectic space

$$
S=S_{-} \oplus S_{+} \text {with }\left[S_{-}: S_{+}\right]=0,
$$

with finite dimension $D=2 \Delta$ and excess $E x=0$ (so $E x_{-}=-E x_{+}$), as in Theorem 4.

A non-zero vector $v \in S$ is separated at the left in case $v \in S_{-} ; v \in S$ is separated at the right in case $v \in S_{+}$; and $v$ is coupled otherwise. If $S_{-}=0$ (or $S_{+}=0$ ), then no such $v$ is coupled.

For any Lagrangian $\Delta$-space $L \subset S$ a basis for this complete Lagrangian $L$ is minimally coupled in case it contains exactly (Nec-coupling $L$ ) vectors, each of which is coupled.

A Lagrangian $\Delta$-space $L \subset S$ is:

strictly separated in case Nec-coupling $L=0$, 
or

$$
\text { totally coupled in case Nec-coupling } L=\Delta \text {. }
$$

The next two corollaries of Theorem 4 clarify the significance of these concepts of separation and coupling, especially with regard to the usage of grade $L$ and Nec-coupling $L$.

Corollary 1. Consider the complex symplectic space

$$
S=S_{-} \oplus S_{+} \text {with }\left[S_{-}: S_{+}\right]=0,
$$

with finite dimension $D=2 \Delta$ and excess $E x=0$ (so $\left.E x_{-}=-E x_{+}\right)$, as in Theorem 4. Let $L$ be a complete Lagrangian subspace in $S$, so $\operatorname{dim} L=\Delta$.

Then $L$ is strictly separated if and only if any one of the following three (logically equivalent) conditions holds:

(i) $\Delta=\operatorname{dim} L \cap S_{-}+\operatorname{dim} L \cap S_{+}$,

(ii) grade $L=0$ and $E x_{ \pm}=0$,

(iii) there exists a basis for $L$ such that each basis vector is separated either at the left (in $S_{-}$) or at the right (in $S_{+}$).

On the other hand, $L$ is totally coupled if and only if any one of the following three (logically equivalent) conditions holds:

(iv) $L \cap S_{-}=L \cap S_{+}=0$,

(v) grade $L=\min \left\{\Delta_{-}, \Delta_{+}\right\}$and $\Delta_{-}=\Delta_{+}$(or alternatively, grade $L=$ $\left.\frac{1}{2}\left(\Delta_{-}+\Delta_{+}\right)\right)$

(vi) for every basis of $L$ each of the basis vectors is coupled.

Proof. First assume that $L$ is strictly separated, so

$$
\text { Nec-coupling } L=\Delta-\operatorname{dim} L \cap S_{-}-\operatorname{dim} L \cap S_{+}=0 \text {, }
$$

which is necessary and sufficient for condition (i). Hence we need only show that conditions (i), (ii), and (iii) are logically equivalent.

But $\Delta=\Delta_{-}+\Delta_{+}+\left|E x_{ \pm}\right|$and $0 \leq \operatorname{dim} L \cap S_{ \pm} \leq \Delta_{ \pm}$, so clearly (i) holds if and only if $\operatorname{dim} L \cap S_{ \pm}=\Delta_{ \pm}$and $E x_{ \pm}=0$. Hence (i) is logically equivalent to (ii).

It is trivial that (i) implies (iii). Conversely, assume (iii), so that there exists a separated basis for $L$, say $\left\{v_{-}^{1}, \ldots, v_{-}^{r}, v_{+}^{1}, \ldots, v_{+}^{s}\right\}$ with $r+s=\Delta$, and the first $r$ vectors lie in $S_{-}$, and the last $s$ vectors lie in $S_{+}$. Suppose $r<\operatorname{dim} L \cap S_{-}$. Then

$$
\Delta=r+s<\operatorname{dim} L \cap S_{-}+\operatorname{dim} L \cap S_{+} \leq \Delta_{-}+\Delta_{+} \leq \Delta,
$$

which is impossible. Hence we conclude that

$$
r=\operatorname{dim} L \cap S_{-} \quad \text { and similarly } \quad s=\operatorname{dim} L \cap S_{+},
$$

so $\Delta=r+s=\operatorname{dim} L \cap S_{-}+\operatorname{dim} L \cap S_{+}$, and condition (i) obtains.

Next assume that $L$ is totally coupled, so

$$
\text { Nec-coupling } L=2 \text { grade } L+\left|E x_{ \pm}\right|=\Delta \text {. }
$$

But this holds if and only if

$$
\left(\Delta_{-}-\operatorname{dim} L \cap S_{-}\right)+\left(\Delta_{+}-\operatorname{dim} L \cap S_{+}\right)+\left|E x_{ \pm}\right|=\Delta_{-}+\Delta_{+}+\left|E x_{ \pm}\right|,
$$

which is necessary and sufficient for the conditions (iv). Hence we need only show that conditions (iv), (v), and (vi) are logically equivalent.

Next we show that (iv) implies (v). Note that if $L \cap S_{-}=L \cap S_{+}=0$, then grade $L=\Delta_{-}=\Delta_{+}$. Conversely, if grade $L=\Delta_{-}=\Delta_{+}$, then we compute that 
Nec-coupling $L=2 \Delta_{ \pm}+\left|E x_{ \pm}\right|=\Delta_{-}+\Delta_{+}+\left|E x_{ \pm}\right|=\Delta$, and hence (v) implies (iv).

For the alternative version of (v), we note that

$$
\text { grade } L=\Delta_{-}=\Delta_{+} \text {implies that grade } L=\frac{1}{2}\left(\Delta_{-}+\Delta_{+}\right) \text {. }
$$

On the other hand, if grade $L=\frac{1}{2}\left(\Delta_{-}+\Delta_{+}\right)$, then 2 grade $L=\Delta_{-}+\Delta_{+}$, and we compute that Nec-coupling $L=\Delta_{-}+\Delta_{+}+\left|E x_{ \pm}\right|=\Delta$. From this we conclude that grade $L=\Delta_{-}=\Delta_{+}$.

Finally, it is trivial that (iv) implies (vi). Conversely, assume (vi), that every basis of $L$ consists of coupled vectors. Then $L \cap S_{-}=L \cap S_{+}=0$, for otherwise there would exist a separated vector in $L$ which could be included in some basis of $L$. Hence (vi) implies (iv).

The next corollary introduces the concept of a minimally coupled basis for the complete Lagrangian $\Delta$-space $L \subset S=S_{-} \oplus S_{+}$, that is, a basis for $L$ which contains exactly (Nec-coupling $L$ ) coupled vectors. Also it is demonstrated that every basis for $L$ can be obtained by a perturbation of a minimally coupled basis.

Corollary 2. Consider the complex symplectic space

$$
S=S_{-} \oplus S_{+}, \quad\left[S_{-}: S_{+}\right]=0,
$$

with finite dimension $D=2 \Delta$ and excess $E x=0$ (so $\left.E x_{-}=-E x_{+}\right)$, as in Theorem 4. Let $L$ be a complete Lagrangian $\Delta$-space in $S$.

Then there exists a minimally coupled basis for $L$, that is, a basis for $L$ containing exactly

Nec-coupling $L=\Delta-\operatorname{dim} L \cap S_{-}-\operatorname{dim} L \cap S_{+}=2$ grade $L+\left|E x_{ \pm}\right|$vectors, each of which is coupled as in Definition 4.

Each minimally coupled basis for $L$ can be shown to contain:

$$
\begin{aligned}
& \text { exactly }\left(\Delta-\operatorname{dim} L \cap S_{-}-\operatorname{dim} L \cap S_{+}\right)=2 \text { grade } L+\left|E x_{ \pm}\right| \\
& \quad \text { vectors, each of which is coupled; } \\
& \text { exactly }\left(\operatorname{dim} L \cap S_{-}\right)=\Delta_{-}-\text {grade } L \text { vectors, each of which } \\
& \quad \text { is separated at the left; } \\
& \text { exactly }\left(\operatorname{dim} L \cap S_{+}\right)=\Delta_{+}-\text {grade } L \text { vectors, each of which } \\
& \quad \text { is separated at the right. }
\end{aligned}
$$

Moreover, each basis of $L$ must definitely contain

at least (Nec-coupling $L$ ) vectors, each coupled,

$$
\begin{aligned}
& \text { at most ( } \left.\operatorname{dim} L \cap S_{-}\right) \text {vectors, each separated at the left, } \\
& \text { at most ( } \left.\operatorname{dim} L \cap S_{+}\right) \text {vectors, each separated at the right. }
\end{aligned}
$$

Furthermore, for each triple of integers $\alpha, \beta$, and $\gamma$ with $0 \leq \alpha \leq \operatorname{dim} L \cap S_{-}$, $0 \leq \beta \leq \operatorname{dim} L \cap S_{+}, \gamma=\Delta-\alpha-\beta$, there exists a basis for $L$ containing exactly

$$
\begin{aligned}
& \gamma \text { vectors, each coupled, } \\
& \alpha \text { vectors, each separated at the left, } \\
& \beta \text { vectors, each separated at the right. }
\end{aligned}
$$

Therefore (2.69) and (2.70) describe all possible bases for L, with regard to separation and coupling. 
Proof. In order to construct a minimally coupled basis for $L$, choose a basis for $L \cap S_{-}$and a basis for $L \cap S_{+}$, and then augment this set of vectors by a further set of $\Delta-\operatorname{dim} L \cap S_{-}-\operatorname{dim} L \cap S_{+}$independent vectors to construct a basis for $L$. Trivially each of these vectors in the augmentation lies neither in $S_{-}$nor in $S_{+}$, and so must be a coupled vector. In this way, we have constructed a minimally coupled basis for $L$.

Indeed, consider any minimally coupled basis for $L$, hence containing exactly

$$
\text { Nec-coupling } L=\Delta-\operatorname{dim} L \cap S_{-}-\operatorname{dim} L \cap S_{+}
$$

coupled vectors. Such a basis of $L$ must then contain exactly (dim $L \cap S_{-}+$ $\operatorname{dim} L \cap S_{+}$) vectors which are each separated. Thus we conclude that the specified basis must contain exactly ( $\operatorname{dim} L \cap S_{-}$) vectors in $S_{-}$, and exactly ( $\operatorname{dim} L \cap S_{+}$) vectors in $S_{+}$, in accord with (2.68) in the corollary.

Now consider an arbitrary basis of $L$. Since the number of basis vectors lying in $S_{-}$is at most ( $\left.\operatorname{dim} L \cap S_{-}\right)$, and the number lying in $S_{+}$is at most $\left(\operatorname{dim} L \cap S_{+}\right)$, then there must be at least $\Delta-\operatorname{dim} L \cap S_{-}-\operatorname{dim} L \cap S_{+}=$Nec-coupling $L$ of the basis vectors that are each coupled, as in (2.69).

Finally take any three integers $\alpha, \beta, \gamma$ such that $0 \leq \alpha \leq \operatorname{dim} L \cap S_{-}, 0 \leq \beta \leq$ $\operatorname{dim} L \cap S_{+}, \gamma=\Delta-\alpha-\beta$. We seek to construct a basis for $L$, containing exactly

$\alpha$ vectors separated at the left,

$\beta$ vectors separated at the right,

and consequently

$\gamma$ vectors that are each coupled.

We begin with a minimally coupled basis for $L$, say

$$
\left\{v_{-}^{1}, \ldots, v_{-}^{a}, v_{+}^{1}, \ldots, v_{+}^{b}, v^{1}, \ldots, v^{c}\right\},
$$

where

$$
a=\operatorname{dim} L \cap S_{-}, \quad b=\operatorname{dim} L \cap S_{+}, \quad c=\Delta-a-b,
$$

and specifically, $v_{-}^{1}, \ldots, v_{-}^{a}$ are each in $S_{-}, v_{+}^{1}, \ldots, v_{+}^{b}$ are each in $S_{+}$, and $v^{1}, \ldots, v^{c}$ are each coupled.

Clearly $\alpha \leq a, \beta \leq b$, and $\gamma \geq c$, with all equalities holding just in case the chosen basis is a minimally coupled basis for $L$. However, suppose $\alpha<a$. In this case we construct a new basis for $L$ upon replacing $v_{-}^{1}$ by the perturbed vector $v_{-}^{1}+\epsilon v^{1}$ for a suitably small $\epsilon>0$ (if $c=0$ replace $v_{-}^{1}$ by $v_{-}^{1}+\epsilon v_{+}^{1}$, and if $b=c=0$ then $\alpha=a=\Delta$, so $\left.S_{+}=0\right)$. Then this new basis contains $(a-1)$ vectors in $S_{-}, b$ vectors in $S_{+}$, and $(c+1)$ coupled vectors.

In this way, the left and right separated vectors can be replaced, one at a time, by routine perturbation techniques of linear algebra, until we obtain the desired basis for $L$, containing exactly $\alpha$ vectors in $S_{-}, \beta$ vectors $S_{+}$, and hence $\gamma$ vectors, each coupled, as in (2.70).

The existence of Lagrangian $\Delta$-spaces with specified grades in the complex symplectic $2 \Delta$-space $S=S_{-} \oplus S_{+}$is demonstrated in the next theorem. These constructions will be carried out in full generality, subject only to the condition that $S$ has excess $E x=0$, which is a necessary and sufficient condition for the existence of any complete Lagrangian subspaces of $S$ (that is, Lagrangian $\Delta$-spaces). 
Theorem 5. Consider a complex symplectic space $S$ with finite dimension $D=2 \Delta$ and excess $E x=0$, and with a prescribed direct sum decomposition

$$
S=S_{-} \oplus S_{+}, \quad\left[S_{-}: S_{+}\right]=0 .
$$

Hence $E x_{-}=-E x_{+}$and $\Delta=\Delta_{-}+\Delta_{+}+\left|E x_{ \pm}\right|$are symplectic invariants, as before.

Then for each integer $\ell=0,1,2, \ldots, \min \left\{\Delta_{-}, \Delta_{+}\right\}$, there exists a complete Lagrangian subspace $L_{\ell}$ with

$$
\text { grade } L_{\ell}=\ell \text {. }
$$

Thus the range of the function $L \rightarrow$ grade $L$ consists of the integers $\ell=0,1,2, \ldots$, $\min \left\{\Delta_{-}, \Delta_{+}\right\}$; and the range of the function $L \rightarrow$ Nec-coupling $L$ consists of the corresponding integers $2 \ell+\left|E x_{ \pm}\right|$, as L runs over the set of all complete Lagrangian spaces in $S$.

Proof. Choose a basis for $S$ so that the corresponding complex skew-Hermitian matrix is the diagonal $2 \Delta \times 2 \Delta$ matrix $\hat{K}$ (illustrated below in (2.72) for the case $\left.E x_{-}=-2\right)$ :

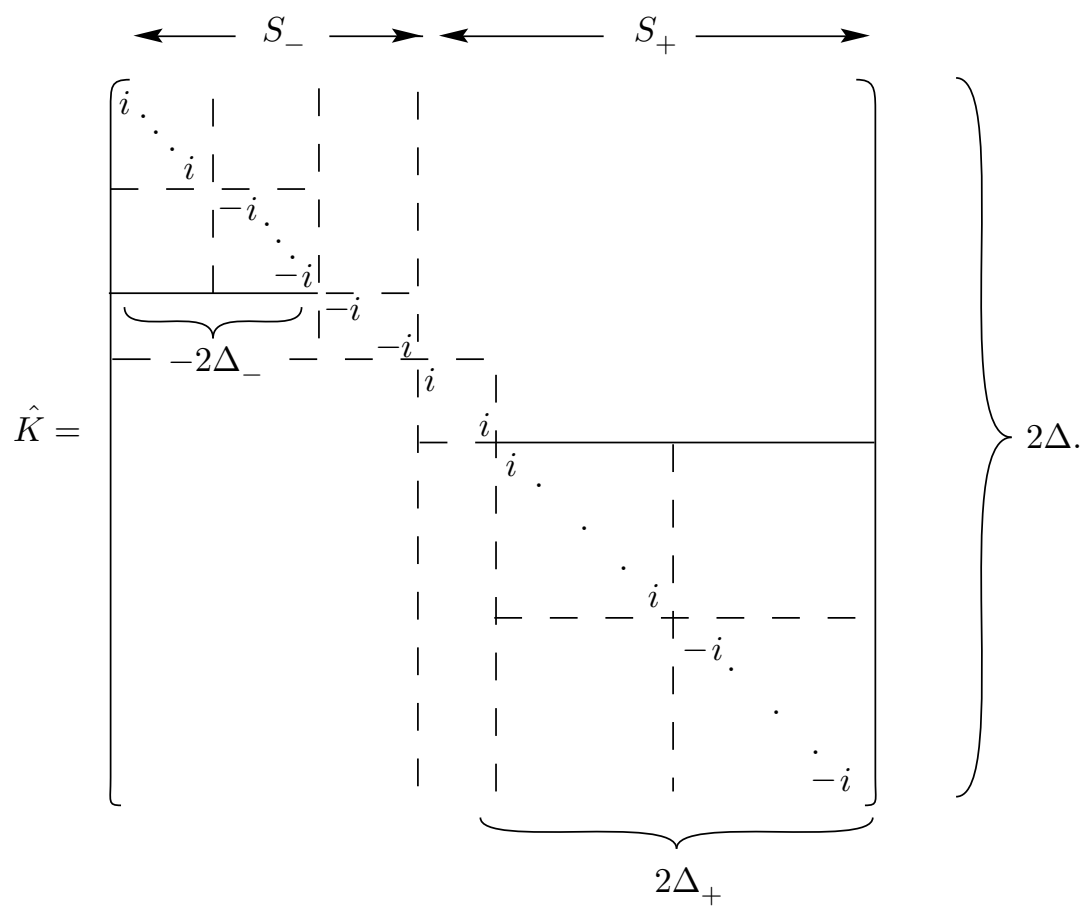

In explicit detail, we label the corresponding basis vectors for $S_{-}$by

$$
e_{+}^{1}, \ldots, e_{+}^{\Delta_{-}}, e_{-}^{1}, \ldots, e_{-}^{\Delta_{-}}, g_{-}^{1}, \ldots, g_{-}^{\left|E x_{-}\right|},
$$

where $e_{+}^{j}$ has +1 in the $j$-place, and otherwise $0 ; e_{-}^{j}$ has +1 in the $\left(\Delta_{-}+j\right)$-place, and otherwise 0 (for $1 \leq j \leq \Delta_{-}$); and $g_{-}^{r}$ has +1 in the $\left(2 \Delta_{-}+r\right.$ )-place, and otherwise 0 (for $1 \leq r \leq\left|E x_{-}\right|$). Further, the corresponding basis vectors for $S_{+}$ are

$$
g_{+}^{1}, \ldots, g_{+}^{\left|E x_{+}\right|}, f_{+}^{1}, \ldots, f_{+}^{\Delta_{+}}, f_{-}^{1}, \ldots, f_{-}^{\Delta_{+}}
$$


Here $g_{+}^{r}$ has +1 in the $\left(2 \Delta_{-}+\left|E x_{-}\right|+r\right)$-place, and otherwise 0 (for $\left.1 \leq r \leq\left|E x_{+}\right|\right)$; and $f_{+}^{k}$ has +1 in the $\left(2 \Delta_{-}+2\left|E x_{ \pm}\right|+k\right)$-place, and otherwise $0 ; f_{-}^{k}$ has +1 in the $\left(2 \Delta_{-}+2\left|E x_{ \pm}\right|+\Delta_{+}+k\right)$-place, and otherwise 0 (for $\left.1 \leq k \leq \Delta_{+}\right)$.

These $2 \Delta_{-}+2 \Delta_{+}+2\left|E x_{ \pm}\right|=2 \Delta$ vectors constitute a basis for $S$, with symplectic products computed by means of the matrix $\hat{K}$ of (2.72). In the case where $\Delta_{-}=0$, or $\Delta_{+}=0$, or $E x_{ \pm}=0$, we omit the corresponding vectors from this basis for $S=S_{-} \oplus S_{+}$.

Then, in accord with the diagonal matrix $\hat{K}$, the symplectic products of these basis vectors can be tabulated:

$$
\begin{aligned}
& {\left[e_{+}^{j}: e_{+}^{j}\right]=i, \quad\left[e_{-}^{j}: e_{-}^{j}\right]=-i \quad \text { for } 1 \leq j \leq \Delta_{-},} \\
& {\left[f_{+}^{k}: f_{+}^{k}\right]=i, \quad\left[f_{-}^{k}: f_{-}^{k}\right]=-i \quad \text { for } 1 \leq k \leq \Delta_{+} \text {, }}
\end{aligned}
$$

and

$$
\left[g_{-}^{r}: g_{-}^{r}\right]=-i, \quad\left[g_{+}^{r}: g_{+}^{r}\right]=i, \quad \text { for } 1 \leq r \leq\left|E x_{ \pm}\right| .
$$

(If $E x_{-}>0$, then these last two symplectic products are $+i$ and $-i$ respectively.)

All the other symplectic products between basis vectors are zero.

Now fix $\ell=0$, and we shall define a basis for the required Lagrangian $\Delta$-space $L_{0}$ which has grade $L_{0}=0$. Namely, take the $\Delta$ independent vectors in $S$,

$$
\begin{gathered}
e_{+}^{1}+e_{-}^{1}, e_{+}^{2}+e_{-}^{2}, \ldots, e_{+}^{\Delta_{-}}+e_{-}^{\Delta_{-}}, \quad \Delta_{-} \text {vectors in } S_{-}, \\
f_{+}^{1}+f_{-}^{1}, f_{+}^{2}+f_{-}^{2}, \ldots, f_{+}^{\Delta_{+}}+f_{-}^{\Delta_{+}}, \quad \Delta_{+} \text {vectors in } S_{+},
\end{gathered}
$$

and

$$
g_{+}^{1}+g_{-}^{1}, g_{+}^{2}+g_{-}^{2}, \ldots, g_{+}^{\left|E x_{ \pm}\right|}+g_{-}^{\left|E x_{ \pm}\right|}, \quad\left|E x_{ \pm}\right| \text {vectors coupled. }
$$

It is clear that $\operatorname{dim} L_{0} \cap S_{-}=\Delta_{-}, \operatorname{dim} L_{0} \cap \Delta_{+}=\Delta_{+}$, so grade $L_{0}=\Delta_{ \pm}-$ $\operatorname{dim} L_{0} \cap S_{ \pm}=0$, as required.

Next take $\ell=1$ and we proceed to modify the prior basis given for $L_{0}$ to obtain a basis for the required Lagrangian $\Delta$-space $L_{1}$ with grade $L_{1}=1$. Namely, delete the two vectors $e_{+}^{1}+e_{-}^{1}$ and $f_{+}^{1}+f_{-}^{1}$, and replace these by the two vectors $e_{+}^{1}+f_{-}^{1}$ and $e_{-}^{1}+f_{+}^{1}$. Then we obtain a new set of $\Delta$ vectors consituting a basis for $L_{1}$ :

$$
\begin{gathered}
e_{+}^{2}+e_{-}^{2}, e_{+}^{3}+e_{-}^{3}, \ldots, e_{+}^{\Delta_{-}}+e_{-}^{\Delta_{-}}, \quad\left(\Delta_{-}-1\right) \text { vectors in } S_{-}, \\
f_{+}^{2}+f_{-}^{2}, f_{+}^{3}+f_{-}^{3}, \ldots, f_{+}^{\Delta_{+}}+f_{-}^{\Delta_{+}}, \quad\left(\Delta_{+}-1\right) \text { vectors in } S_{+},
\end{gathered}
$$

and

$$
g_{+}^{1}+g_{-}^{1}, \ldots, g_{+}^{\left|E x_{ \pm}\right|}+g_{-}^{\left|E x_{ \pm}\right|}, e_{+}^{1}+f_{-}^{1}, e_{-}^{1}+f_{+}^{1},
$$

a set of $\left(\left|E x_{ \pm}\right|+2\right)$ vectors each of which lies neither in $S_{-}$nor in $S_{+}$, and hence each of which is coupled.

It is clear that $\operatorname{dim} L_{1} \cap S_{-}=\Delta_{-}-1, \operatorname{dim} L_{1} \cap S_{+}=\Delta_{+}-1$, so that grade $L_{1}=\Delta_{ \pm}-\operatorname{dim} L_{1} \cap S_{ \pm}=1$, as required.

In the next step, take $\ell=2$ and then modify the prior basis for $L_{1}$ to obtain a new set of $\Delta$ vectors that will constitute a basis for the Lagrangian $\Delta$-space $L_{2}$ with grade $L_{2}=2$. Namely, delete the two vectors $e_{+}^{2}+e_{-}^{2}$ and $f_{+}^{2}+f_{-}^{2}$, and replace these by the two vectors $e_{+}^{2}+f_{-}^{2}$ and $e_{-}^{2}+f_{+}^{2}$. It is easy to verify that $\operatorname{dim} L_{2} \cap S_{ \pm}=\Delta_{ \pm}-2$, and thus grade $L_{2}=2$. 
Continue this process for $\ell=3,4, \ldots, \min \left\{\Delta_{-}, \Delta_{+}\right\}$. For simplicity of exposition assume that $\Delta_{+} \geq \Delta_{-}$, and then the basis for $L_{\Delta_{-}}$is given by the $\Delta$ vectors

$$
f_{+}^{\Delta_{-}+1}+f_{-}^{\Delta_{-}+1}, \ldots, f_{+}^{\Delta_{+}}+f_{-}^{\Delta_{+}}, \quad\left(\Delta_{+}-\Delta_{-}\right) \text {vectors in } S_{+}
$$

(omit these vectors if $\Delta_{+}=\Delta_{-}$, and replace by corresponding vectors from $S_{-}$if $\left.\Delta_{+}<\Delta_{-}\right)$, and then $\left(\left|E x_{ \pm}\right|+2 \Delta_{-}\right)$vectors are each coupled, namely,

$$
g_{+}^{1}+g_{-}^{1}, \ldots, g_{+}^{\left|E x_{ \pm}\right|}+g_{-}^{\left|E x_{ \pm}\right|}, e_{+}^{1}+f_{-}^{1}, e_{-}^{1}+f_{+}^{1}, \ldots, e_{+}^{\Delta_{-}}+f_{-}^{\Delta_{-}}, e_{-}^{\Delta_{-}}+f_{+}^{\Delta_{-}} .
$$

Then $\operatorname{dim} L_{\Delta_{-}} \cap S_{-}=0, \operatorname{dim} L_{\Delta_{-}} \cap S_{+}=\Delta_{+} \Delta_{-}$, so grade $L_{\Delta_{-}}=\Delta_{-}$.

Corollary 1. For each prescribed integer $\ell=0,1,2, \ldots, \min \left\{\Delta_{-}, \Delta_{+}\right\}$, there exists a complete Lagrangian subspace $L_{\ell}$, with grade $L_{\ell}=\ell$, in the complex symplectic $2 \Delta$-space $S=S_{-} \oplus S_{+}$having $E x=0$, as in Theorem 5 .

Moreover, there then exists a minimally coupled basis for $L_{\ell}$ containing exactly

$$
\text { Nec-coupling } L_{\ell}=2 \ell+\left|E x_{ \pm}\right| \text {vectors, each coupled, }
$$

and also

$$
\operatorname{dim} L_{\ell} \cap S_{-}=\Delta_{-}-\ell \text { vectors, each separated at the left, }
$$

and

$\operatorname{dim} L_{\ell} \cap S_{+}=\Delta_{+}-\ell$ vectors, each separated at the right.

\section{Appendix: Applications to the THEORY of SELF-ADJOINT OPERATORS}

Let $H$ be a complex Hilbert space (finite or infinite dimensional of any cardinality), with Hermitian inner product $\langle\cdot, \cdot\rangle$, and corresponding complete norm $\|\cdot\|$ as usual. Let $T_{1}$ be a (linear) operator in $H$, with a (linear manifold) domain $D\left(T_{1}\right) \subseteq H$. Then define the skew-Hermitian sesquilinear form [:] on $D\left(T_{1}\right) \times D\left(T_{1}\right) \rightarrow \mathbf{C}$, by

$$
f, g \rightarrow[f: g] \equiv\left\langle T_{1} f, g\right\rangle-\left\langle f, T_{1} g\right\rangle, \text { for } f, g \in D\left(T_{1}\right) .
$$

Next define the operator $T_{0}$ on $D\left(T_{0}\right) \subseteq D\left(T_{1}\right)$ by

(i) $\quad D\left(T_{0}\right) \equiv\left\{f \in D\left(T_{1}\right) \mid\left[f: D\left(T_{1}\right)\right] \equiv 0\right\}$,

$$
\text { (ii) } \quad T_{0} f=T_{1} f \text { for } f \in D\left(T_{0}\right) \text {. }
$$

Thus

$$
T_{0} \subseteq T_{1}, D\left(T_{0}\right) \subseteq D\left(T_{1}\right),
$$

so $T_{0}$ is the restriction of $T_{1}$ to the domain $D\left(T_{0}\right)$.

Now assume the following properties of these two operators $T_{0}$ and $T_{1}$ in $H$ :

(i) $D\left(T_{0}\right)$ is dense in $H$, so also $D\left(T_{1}\right)$ is dense in $H$,

$$
\text { (ii) } \quad \text { adjoints } T_{0}^{*}=T_{1} \text { and } T_{1}^{*}=T_{0} \text {, }
$$

so both $T_{0}$ and $T_{1}$ are closed operators. In fact, $T_{0}$ is a closed operator with dense domain $D\left(T_{0}\right) \subseteq H$, and is symmetric, that is,

$$
[f: g]=0 \quad \text { for } f, g \in D\left(T_{0}\right) \text {. }
$$

Note that the second condition in (A.4) (ii) is redundant, because $T_{0}^{* *}=T_{0}$ from general theory [DS]. 
Remarks on basic properties of adjoints. Let $T$ be any linear operator with a dense domain $D(T) \subseteq H$. Then the adjoint operator $T^{*}$ is defined as follows: $f \in D\left(T^{*}\right) \subseteq H$ if and only if there exists some $f^{*} \in H$ such that

$$
\left\langle f^{*}, g\right\rangle=\langle f, T g\rangle, \quad \text { for all } g \in D(T) .
$$

Then $f^{*}$, depending on $f$, is unique since $D(T)$ is dense in $H$. We define $T^{*} f=f^{*}$ for $f \in D\left(T^{*}\right)$, and $T^{*}$ is thus a linear operator with domain $D\left(T^{*}\right) \subseteq H$; in fact, $T^{*}$ is a closed operator in $H$, see [DS]. Accordingly, we write

$$
\left\langle T^{*} f, g\right\rangle=\langle f, T g\rangle \text {, for all } f \in D\left(T^{*}\right), g \in D(T) .
$$

Clearly, if $V$ is another linear operator on $D(V) \subseteq H$, and if $T \subseteq V$ on $D(T) \subseteq$ $D(V)$ (so $T$ is a restriction of $V$ to the domain $D(T)$ ), then $V^{*} \subseteq T^{*}$ on $D\left(V^{*}\right) \subseteq$ $D\left(T^{*}\right)$, so $V^{*}$ is a restriction of $T^{*}$ to the domain $D\left(V^{*}\right)$.

We further define the operator $T$ on the dense domain $D(T) \subseteq H$ to be selfadjoint in case

$$
T=T^{*} \text { on } D(T)=D\left(T^{*}\right) .
$$

We are interested in operators $T$ on $D(T) \subseteq H$ which are self-adjoint extensions of the given operator $T_{0}$ on $D\left(T_{0}\right)$, that is, (A.6) obtains and

$$
T_{0} \subseteq T \text { on } D\left(T_{0}\right) \subseteq D(T)
$$

(so $T_{0}$ is the restriction of $T$ to the domain $D\left(T_{0}\right)$ ). Then $T=T^{*}$ is a closed operator on the dense domain $D(T)=D\left(T^{*}\right) \subseteq H$, and by (A.5) $T$ is symmetric

$$
[f: g]=\langle T f, g\rangle-\langle f, T g\rangle=0, \quad \text { for } f, g \in D(T) .
$$

In the case when the given operators $T_{0}$ and $T_{1}$ satisfy the conditions (A.4) (i) and (ii), we note that each self-adjoint operator $T$ on $D(T) \subseteq H$ which is an extension of $T_{0}$ (that is, $T_{0}$ is a the restriction of $T$ to the domain $D\left(T_{0}\right) \subseteq D(T)$ ) satisfies

$$
T=T^{*} \subseteq T_{0}^{*}=T_{1} \text {, so } D(T)=D\left(T^{*}\right) \subseteq D\left(T_{1}\right),
$$

and hence both $T_{0}$ and $T$ are restrictions of $T_{1}$. We summarize this accordingly:

$$
T_{0} \subseteq T \subseteq T_{1} \text { on } D\left(T_{0}\right) \subseteq D(T) \subseteq D\left(T_{1}\right)
$$

for each self-adjoint extension $T$ of $T_{0}$, see [DS], [NA].

Definition 1. Consider the quotient (or identification) complex vector space

$$
\mathbf{S}=D\left(T_{1}\right) / D\left(T_{0}\right),(\operatorname{dim} \mathbf{S}=\infty \text { allowed }),
$$

arising from the operators $T_{0} \subseteq T_{1}$ on $D\left(T_{0}\right) \subseteq D\left(T_{1}\right) \subseteq H$, satisfying (A.2), (A.3), (A.4) above. Each vector of $\mathbf{S}$ is a coset of some function $f \in D\left(T_{1}\right)$, and we write

$$
\mathbf{f}=\left\{f+D\left(T_{0}\right)\right\} \in \mathbf{S} .
$$

Define the skew-Hermitian sesquilinear form on $\mathbf{S}$,

$$
\mathbf{f}, \mathbf{g} \rightarrow[\mathbf{f}: \mathbf{g}], \mathbf{S} \times \mathbf{S} \rightarrow \mathbf{C},
$$

by

$$
[\mathbf{f}: \mathbf{g}]=\left[f+D\left(T_{0}\right): g+D\left(T_{0}\right)\right]:=[f: g],
$$

for any functions $f, g \in D\left(T_{1}\right)$ representing $\mathbf{f}, \mathbf{g}$, respectively. 
Because of (A.2), this skew-Hermitian form on $\mathbf{S}$ is well-defined (does not depend on the choice of the coset representatives), and we use the same notation as for the corresponding form of (A.1) on $D\left(T_{1}\right)$, with no ambiguity. However, we use the bold-face notation $\mathbf{f}, \mathbf{S}$ and (later) $\mathbf{L}$ to emphasize that these are cosets, or collections of cosets.

Lemma 1. The complex vector space

$$
\mathbf{S}=D\left(T_{1}\right) / D\left(T_{0}\right),
$$

with the skew-Hermitian form [:], as in Definition 1, is a complex symplectic space (see Section 1, (1.1)).

Proof. The form $\mathbf{f}, \mathbf{g} \rightarrow[\mathbf{f}: \mathbf{g}] \in \mathbf{C}$ is clearly skew-Hermitian and sesquilinear on $\mathbf{S}$ (properties inherited from the corresponding form (A.1) on $D\left(T_{1}\right)$ ). Also it is non-degenerate because

$$
[\mathbf{f}: \mathbf{S}]=0 \text { implies }\left[f: D\left(T_{1}\right)\right]=0,
$$

so then $f \in D\left(T_{0}\right)$ and $\mathbf{f}=0$ in $\mathbf{S}$.

The following theorem implies the GKN-theorem (see Section 1, Example 3) concerning the boundary value problem for Lagrange symmetric (i.e. formally self-adjoint) linear ordinary differential (or quasi-differential [EM], [EV], [EZ]) expressions on arbitrary real intervals. We shall precisely phrase the GKN-theorem, and provide its proof, later as a corollary to our Theorem 1 below.

We recall from Section 1, Definition 2 that a linear submanifold $\mathbf{L} \subset \mathbf{S}$ is Lagrangian in case $[\mathbf{L}: \mathbf{L}]=0$, and furthermore $\mathbf{L}$ is complete in case:

$$
\mathbf{f} \in \mathbf{S} \text { and }[\mathbf{f}: \mathbf{L}]=0 \text { imply } \mathbf{f} \in \mathbf{L} \text {. }
$$

Since, at this stage there is no specified topology imposed on $\mathbf{S}$, we shall often use the terminology of linear subspaces rather than linear submanifolds in $\mathbf{S}$. We offer some additional comments on topologies in $\mathbf{S}$ immediately after the proof of Theorem 1.

Theorem 1. Consider linear operators $T_{0} \subseteq T_{1}$ on domains $D\left(T_{0}\right) \subseteq D\left(T_{1}\right)$ in the complex Hilbert space $H$, satisfying conditions (A.2), (A.3), (A.4) above. Then

$$
\mathbf{S}=D\left(T_{1}\right) / D\left(T_{0}\right),
$$

with the symplectic form [:] of Definition 1, is a complex symplectic space.

Under these circumstances there exists a natural bi-unique correspondence between the set $\{T\}$ of all self-adjoint extensions $T$ of $T_{0}$, and the set $\{\mathbf{L}\}$ of all complete Lagrangian subspaces $\mathbf{L}$ of $\mathbf{S}$. Namely, for each such self-adjoint operator $T$ on $D(T)$ of $T_{0}$ on $D\left(T_{0}\right)$, the corresponding complete Lagrangian subspace $\mathbf{L} \subset \mathbf{S}$ is

$$
\mathbf{L}=D(T) / D\left(T_{0}\right) .
$$

In particular, there exists such a self-adjoint extension $T$ of $T_{0}$ if and only if there exists a complete Lagrangian subspace $\mathbf{L}$ of $\mathbf{S}$. The case where $T_{0}=T_{1}$ arises if and only if $T_{0}=T_{0}^{*}$ is the unique such self-adjoint extension of $T_{0}$, and this occurs if and only if $\mathbf{S}=0$. 
Proof. Take any self-adjoint operator $T$ on $D(T)$, so

$$
T_{0} \subseteq T=T^{*} \subseteq T_{1} \text { on } D\left(T_{0}\right) \subseteq D(T)=D\left(T^{*}\right) \subseteq D\left(T_{1}\right),
$$

so $T_{0}$ and $T$ are restrictions of $T_{1}$ to $D\left(T_{0}\right)$ and $D(T)$, respectively, as in (A.8). Then define the following subset of $\mathbf{S}$ :

$$
\mathbf{L}=D(T) / D\left(T_{0}\right),
$$

that is, $\mathbf{L}$ consists of all the cosets $\mathbf{f}=\left\{f+D\left(T_{0}\right)\right\}$ with $f \in D(T)$.

We must verify that $\mathbf{L}$ is a complete Lagrangian subspace of the complex symplectic space $\mathbf{S}=D\left(T_{1}\right) / D\left(T_{0}\right)$ of (A.12). Clearly $\mathbf{L}$ is a linear subspace of $\mathbf{S}$, since $\mathbf{L}$ is the image of the linear manifold $D(T) \subseteq D\left(T_{1}\right)$ under the natural projection map

$$
\Psi: D\left(T_{1}\right) \rightarrow \mathbf{S}, f \rightarrow \mathbf{f}=\left\{f+D\left(T_{0}\right)\right\} .
$$

We next calculate, for $\mathbf{f}=\left\{f+D\left(T_{0}\right)\right\}$ and $\mathbf{g}=\left\{g+D\left(T_{0}\right)\right\}$ where $f, g \in D(T)$,

$$
\begin{aligned}
{[\mathbf{f}: \mathbf{g}]=[f: g] } & =\left\langle T_{1} f, g\right\rangle-\left\langle f, T_{1} g\right\rangle \\
& =\langle T f, g\rangle-\langle f, T g\rangle=0,
\end{aligned}
$$

since $T$ is symmetric on its domain $D(T)$. Hence $\mathbf{L}$ is a Lagrangian subspace of $\mathbf{S}$.

In order to show that $\mathbf{L}$ is a complete Lagrangian, take any $h \in D\left(T_{1}\right)$ for which

$$
[\mathbf{h}: \mathbf{L}]=0 \text {, or }[h: g]=0 \text { for all } g \in D(T) .
$$

Then

$$
\left\langle T_{1} h, g\right\rangle=\left\langle h, T_{1} g\right\rangle=\langle h, T g\rangle, \text { for all } g \in D(T) .
$$

But this means that $h \in D\left(T^{*}\right)=D(T)$, and so $\mathbf{h} \in \mathbf{L}$. Therefore $\mathbf{L}$ is a complete Lagrangian subspace of $\mathbf{S}$.

On the other hand, we now let $\mathbf{L}$ be any chosen complete Lagrangian subspace of $\mathbf{S}$, and we seek to define a corresponding self-adjoint extension $T$ of $T_{0}$ whose domain $D(T)$ projects onto $\mathbf{L}$, that is, we require that $\Psi D(T)=\mathbf{L}$. Accordingly, define the set $D(T) \subseteq D\left(T_{1}\right)$ by the set mapping $\Psi^{-1}$, the inverse set mapping of $\Psi$ in (A.16),

$$
D(T) \equiv \Psi^{-1} \mathbf{L}=\left\{h \in D\left(T_{1}\right) \mid \mathbf{h} \in \mathbf{L}\right\} .
$$

Then $D(T)$ is clearly a linear submanifold of $H$, and

$$
D\left(T_{0}\right) \subseteq D(T) \subseteq D\left(T_{1}\right),
$$

and we define the operator $T$ as the restriction of $T_{1}$ to the domain $D(T)$. We must verify that $T$ on $D(T)$ is self-adjoint, and furthermore that $\mathbf{L}=D(T) / D\left(T_{0}\right)$.

Recall that $f \in D\left(T^{*}\right)$ in case there exists some $f^{*} \in H$ for which $\left\langle f^{*}, g\right\rangle=$ $\langle f, T g\rangle$, for all $g \in D(T)$. Also note that $\langle f, T g\rangle=\left\langle f, T_{1} g\right\rangle$ for $g \in D(T)$.

Since $\mathbf{L}$ is a Lagrangian subspace of $\mathbf{S}$,

$$
[f: g]=\langle T f, g\rangle-\langle f, T g\rangle=0, \text { for all } g \in D(T),
$$

whenever $f \in D(T)$. Thus we see that $D(T) \subseteq D\left(T^{*}\right)$, and so $T$ is a symmetric operator.

Furthermore, since

$$
T_{0} \subseteq T \subseteq T_{1} \text { on } D\left(T_{0}\right) \subseteq D(T) \subseteq D\left(T_{1}\right)
$$


(and both $T_{0}$ and $T$ are restrictions of the operator $T_{1}$ ), we note that

$$
T_{1}^{*} \subseteq T^{*} \subseteq T_{0}^{*} \text { on } D\left(T_{1}^{*}\right) \subseteq D\left(T^{*}\right) \subseteq D\left(T_{0}^{*}\right)
$$

or

$$
T_{0} \subseteq T^{*} \subseteq T_{1} \text { on } D\left(T_{0}\right) \subseteq D\left(T^{*}\right) \subseteq D\left(T_{1}\right),
$$

so $T^{*}$ is also a restriction of $T_{0}^{*}=T_{1}$.

Now fix any $f \in D\left(T^{*}\right)$ and compute

$$
\left\langle T^{*} f, g\right\rangle=\langle f, T g\rangle, \text { for all } g \in D(T),
$$

so

$$
\left\langle T_{1} f, g\right\rangle=\left\langle f, T_{1} g\right\rangle, \text { for all } g \in D(T) .
$$

Hence we conclude that

$$
[\mathbf{f}: \mathbf{g}]=[f: g]=0, \text { and thus }[\mathbf{f}: \mathbf{L}]=0 .
$$

But since $\mathbf{L}$ is complete, $\mathbf{f} \in \mathbf{L}$, and so $f \in D(T)$. Therefore $D\left(T^{*}\right) \subseteq D(T)$, so $D\left(T^{*}\right)=D(T)$, as required. We have verified that $T=T^{*}$ (both restrictions of $T_{1}$ ) on their common domain $D(T)=D\left(T^{*}\right)$. Therefore the operator $T$ is a self-adjoint extension of $T_{0}$.

But trivially, $\Psi D(T)=\mathbf{L}$, and hence $\mathbf{L}=D(T) / D\left(T_{0}\right)$, as required.

Hence the map induced by $\Psi$, as in (A.16), is

$$
\boldsymbol{\Psi}:\{T\} \rightarrow\{\mathbf{L}\} \text {, defined by } T \rightarrow \mathbf{L}=D(T) / D\left(T_{0}\right),
$$

which is surjective.

Finally, we show that $\boldsymbol{\Psi}$ in (A.21) is injective. Take two different self-adjoint operators $T_{\alpha} \neq T_{\beta}$ (both extensions of $T_{0}$ on $D\left(T_{0}\right)$, and hence restrictions of $\left.T_{0}^{*}=T_{1}\right)$, with corresponding domains $D\left(T_{\alpha}\right)$ and $D\left(T_{\beta}\right) \subseteq D\left(T_{1}\right)$. Since the two operators $T_{\alpha}$ and $T_{\beta}$ are different, this implies that $D\left(T_{\alpha}\right) \neq D\left(T_{\beta}\right)$, and so there exists an element $u \in D\left(T_{\alpha}\right)$ but $u \neq D\left(T_{\beta}\right)$ (or vice versa). In particular, $u \notin D\left(T_{0}\right)$. Then $\mathbf{u}=\left\{u+D\left(T_{0}\right)\right\}$ satisfies

$$
\mathbf{u} \in \mathbf{L}_{\alpha}=D\left(T_{\alpha}\right) / D\left(T_{0}\right), \text { but } \mathbf{u} \notin \mathbf{L}_{\beta}=D\left(T_{\beta}\right) / D\left(T_{0}\right)
$$

(for this would mean that all $\left\{u+D\left(T_{0}\right)\right\} \subseteq D\left(T_{\beta}\right)$, contradicting $u \notin D\left(T_{\beta}\right)$ ).

We have demonstrated that $T_{\alpha} \neq T_{\beta}$ implies that $\mathbf{L}_{\alpha} \neq \mathbf{L}_{\beta}$, and therefore the map $\boldsymbol{\Psi}$ of (A.21) is an injection onto $\{\mathbf{L}\}$. Therefore $\boldsymbol{\Psi}$ defines the required bijective correspondence of $\{T\}$ with $\{\mathbf{L}\}$, as asserted in the theorem.

Remarks on topologies for complex symplectic spaces. Let $S$ be an abstract complex symplectic space, with symplectic form [:], as in Definition 1 in Section 1 above. If $S$ has finite dimension, $\operatorname{dim} S=D$, then $S$ is linearly isomorphic with the complex number space $\mathbf{C}^{D}$, and we use the standard topology of $\mathbf{C}^{D}$ on $S$. In this case all the algebraic operations in $S$ are continuous; in particular the map

$$
u, v \rightarrow[u: v], \quad S \times S \rightarrow \mathbf{C}
$$

is continuous (jointly) in both variables $u, v \in S$.

However, if $\operatorname{dim} S=\infty$ then various possibilities arise. For example, consider, as in Section 1 above,

$$
S=H_{-} \oplus H_{+} \text {with }\left[H_{-}: H_{+}\right]=0,
$$


where $H_{ \pm}$are each complex Hilbert spaces with corresponding Hermitian inner products $\langle\cdot, \cdot\rangle_{ \pm}$. Furthermore assume the corresponding unique decompositions $u=u_{-}+u_{+}$and $v=v_{-}+v_{+}$, for vectors $u, v \in S$, satisfy

$$
[u: v]=-i\left\langle u_{-}, v_{-}\right\rangle_{-}+i\left\langle u_{+}, v_{+}\right\rangle_{+},
$$

in terms of the given symplectic form [:] on $S$, as in Theorem 3 of Section 1 . Then we can topologize $S$ as a complete metric space by the norm $\|\cdot\|$,

$$
\|u\|^{2}=\left\langle u_{-}, u_{-}\right\rangle_{-}+\left\langle u_{+}, u_{+}\right\rangle_{+},
$$

so that $S$ is the Hilbert space direct sum of $H_{-}$and $H_{+}$. In this case, we again find that the map

$$
u, v \rightarrow[u: v], S \times S \rightarrow \mathbf{C}
$$

is (jointly) continuous.

As an important special case illustrating this construction, consider $\mathbf{S}=$ $D\left(T_{1}\right) / D\left(T_{0}\right)$, where $T_{1}$ on $D\left(T_{1}\right)$ and $T_{0}$ on $D\left(T_{0}\right)$ are operators on the Hilbert space $H$, as in Theorem 1 above. Then introduce the $T_{1}$-graph norm $\|\cdot\|_{1}$ on $D\left(T_{1}\right)$, as in [DS],

$$
\|f\|_{1}^{2}=\langle f, f\rangle+\left\langle T_{1} f, T_{1} f\right\rangle, \quad \text { for } f \in D\left(T_{1}\right),
$$

so that $D\left(T_{1}\right)$ becomes a complex Hilbert space with the Hilbert subspace $D\left(T_{0}\right)$. Then $\mathbf{S}$ is also a Hilbert space, considered as the orthocomplement of $D\left(T_{0}\right)$ in $D\left(T_{1}\right)$ (see [DS, XII 4.10]), and furthermore $u, v \rightarrow[u: v]$ is a continuous map of $\mathbf{S} \times \mathbf{S} \rightarrow \mathbf{C}$.

In the most general case for $\operatorname{dim} S$ infinite, we can define a topology on $S$ by requiring that

$$
u \rightarrow\left[u: v_{0}\right], S \rightarrow \mathbf{C} \quad\left(\text { fixed } v_{0} \in S\right)
$$

is continuous, for each choice of $v_{0} \in S$. That is, for each $v_{0} \in S$ and open set $\mathcal{O} \subset \mathbf{C}$, define the open set in $S$,

$$
\left\{u \in S \mid\left[u: v_{0}\right] \subset \mathcal{O}\right\},
$$

and use these sets as a subbase for the corresponding weak topology on $S$.

In this case all the maps of $S \rightarrow \mathbf{C}$

$$
u \rightarrow[u: v] \text { and } u \rightarrow[v: u]=-\overline{[u: v]}
$$

are continuous for each fixed $v \in S$.

Proposition. Let $S$ be a complex symplectic space, with symplectic form [:], and assume that there is given a topology on $S$ for which all the maps indicated in (A.22) are continuous.

Then each complete Lagrangian submanifold $L \subset S$ is a closed set in $S$.

Proof. Let $L$ be a complete Lagrangian submanifold in $S$; we show that the complement of $L$ is open in the given topology of $S$.

Take a point $u_{0} \in S$ in the complement of $L$. Then $\left[u_{0}: L\right]$ is not zero, for otherwise $u_{0} \in L$. That is, there exists some point $v_{0} \in L$ with $\left[u_{0}: v_{0}\right] \neq 0$. Then define the open subset of $S$

$$
U=\left\{u \in S||\left[u: v_{0}\right]\left|>\frac{1}{2}\right|\left[u_{0}: v_{0}\right] \mid\right\}
$$


(note: $|z|>\frac{1}{2}\left|\left[u_{0}: v_{0}\right]\right|$ defines an open subset of $\mathbf{C}$ ). Thus $U$ is an open neighborhood of $u_{0}$, yet $U \cap L=\emptyset$.

We close this Appendix with a brief introduction to the applications of Theorem 1 to the boundary value problems for Lagrange-symmetric (formally self-adjoint) linear ordinary differential expressions $M$ of arbitrary order $n \geq 1$, on arbitrary real (non-degenerate) intervals $\mathcal{J} \subset \mathbf{R}$ (open, closed, half-closed, compact or infinitebut containing an interior point). Thus consider the linear expression (or operator)

$$
M[y]=p_{n} y^{(n)}+p_{n-1} y^{(n-1)}+\cdots+p_{1} y^{\prime}+p_{0} y
$$

on the non-degenerate interval $\mathcal{J}$, with endpoints $-\infty \leq a, b \leq+\infty$, where the complex-valued coefficients $p_{j} \in L_{l o c}^{1}(\mathcal{J})$ for $j=0,1,2, \ldots, n-1$, and further $p_{n} \in A C_{\text {loc }}(\mathcal{J})$ and $p_{n}(x) \neq 0$ for all $x \in \mathcal{J}$. The corresponding domain for $M$ is

$$
D(M) \equiv\left\{y: \mathcal{J} \rightarrow \mathbf{C} \mid y^{(r)} \in A C_{l o c}(\mathcal{J}) \text { for } r=0,1, \ldots, n-1\right\}
$$

in terms of the ordinary derivatives $y^{(r)}$, so here $y^{(n)}$ and also $M[y] \in L_{l o c}^{1}(\mathcal{J})$.

There is an important special case where $M$ has smooth coefficients $p_{j} \in C^{j}(\mathcal{J})$, or even $p_{j} \in C^{\infty}(\mathcal{J})$ for $j=0,1, \ldots, n$ (where $C^{k}(\mathcal{J}), 0 \leq k \leq \infty$, consists of all complex-valued functions with $k$ continuous derivatives on some open neighborhood of $\mathcal{J}$, as usual). In these cases the classical condition of Lagrange provides a direct method of verifying the Lagrange symmetry of such a differential expression $M$. However, even in the general case (A.23), we can define $M$ to be Lagrangesymmetric (or formally self-adjoint) on $\mathcal{J}$ when

$$
\int_{a}^{b}\{M[f] \bar{g}-f \overline{M[g]}\} d x=0,
$$

for all functions $f, g \in D(M)$ with compact supports interior to $\mathcal{J}$.

For such a Lagrange-symmetric differential expression $M$ on $\mathcal{J}$, following the notations of Example 3 in Section 1, we define two operators $T_{0}$ on $D\left(T_{0}\right)$ and $T_{1}$ on $D\left(T_{1}\right)$ in the complex Hilbert space $H=L^{2}(\mathcal{J})$. Namely, define $T_{1}$ as the "maximal operator"

$$
T_{1} f=M[f] \text { on } D\left(T_{1}\right)=\left\{f \in D(M) \mid f \text { and } M[f] \in L^{2}(\mathcal{J})\right\}
$$

and $T_{0}$ as the "minimal operator"

$$
T_{0} f=M[f] \text { on } D\left(T_{0}\right)=\left\{f \in D\left(T_{1}\right) \mid\left[f: D\left(T_{1}\right)\right]=0\right\} .
$$

Here the skew-Hermitian form [:] on $D\left(T_{1}\right)$ is given by

$$
[f: g]=\left\langle T_{1} f, g\right\rangle-\left\langle f, T_{1} g\right\rangle, \text { for } f, g \in D\left(T_{1}\right) .
$$

It is known [AG], [EZ], [NA] that $T_{0} \subseteq T_{1}$ on $D\left(T_{0}\right) \subseteq D\left(T_{1}\right) \subset H$ satisfy the fundamental hypothesis (A.4).

As before in this Appendix, we define the endpoint space $\mathbf{S}$, for $M$ on $\mathcal{J}$, as the quotient or identification vector space

$$
\mathbf{S}=D\left(T_{1}\right) / D\left(T_{0}\right),
$$

so there is then a natural projection map

$$
\Psi: D\left(T_{1}\right) \rightarrow \mathbf{S}, f \rightarrow \mathbf{f}=\left\{f+D\left(T_{0}\right)\right\}, \text { for } f \in D\left(T_{1}\right) .
$$


Then it is easy to verify, as in Lemma 1 of Theorem 1, that $\mathbf{S}$ in (A.29), with the symplectic form [:] inherited from $D\left(T_{1}\right)$ in (A.28), is a complex symplectic space where the symplectic product of $\mathbf{f}=\left\{f+D\left(T_{0}\right)\right\}$ and $\mathbf{g}=\left\{g+D\left(T_{0}\right)\right\}$ is given by

$$
[\mathbf{f}: \mathbf{g}]:=[f: g] \text {. }
$$

As before, we use the same notation [:] in both $D\left(T_{1}\right)$ and $\mathbf{S}$.

Since the operators $T_{1}$ and $T_{0}$ arise from the differential expression $M$ of order $n \geq 1$, it follows from the theory of linear ordinary differential equations [DS] that $\mathbf{S}$ has a finite dimension

$$
\operatorname{dim} \mathbf{S} \leq 2 n
$$

Moreover, the symplectic invariants of $\mathbf{S}$ are related to the deficiency indices $d^{ \pm}$of $M$ on $\mathcal{J}$ (see $[\mathrm{DS}],[\mathrm{EI}])$, by

$$
\begin{aligned}
p & =d^{+} \quad(\text { positivity index } \mathbf{S}), \\
q & =d^{-} \quad(\text { negativity index } \mathbf{S}), \\
\operatorname{dim} \mathbf{S} & =p+q=d^{+}+d^{-} \quad(\operatorname{dimension} \mathbf{S}), \\
E x & =p-q=d^{+}-d^{-} \quad(\operatorname{excess} \mathbf{S}) .
\end{aligned}
$$

Hence the excess $E x=0$ if and only if $d^{+}=d^{-}$(which we then denote by the non-negative integer $d=d^{ \pm}$), and in such a case $\operatorname{dim} \mathbf{S}=2 d$, see [EI], [EM]. In the "regular problem" where $\mathcal{J}=[a, b]$ is compact, it is always the case that $d^{+}=d^{-}=n$; but in the "singular problem" [TU], it may or may not be the case that $d^{+}=d^{-}$or $E x=0$. We recall that a finite dimensional complex symplectic space has a complete Lagrangian subspace if and only if $E x=0$, according to Theorem 2 of Section 2 above.

We can now state and prove the GKN-theorem for the symmetric differential expression $M$ on the interval $\mathcal{J}$, and we present this important result as a corollary of Theorem 1 above.

Corollary 1 (GKN-Theorem). Consider the Lagrange-symmetric linear differential expression $M$ of (A.23) of order $n \geq 1$ on the non-degenerate real interval $\mathcal{J}$. Let $T_{0}$ on $D\left(T_{0}\right)$ and $T_{1}$ on $D\left(T_{1}\right)$ be the minimal and maximal operators, respectively, as generated by $M$ in the complex Hilbert space $L^{2}(\mathcal{J})$, and let $\mathbf{S}=D\left(T_{1}\right) / D\left(T_{0}\right)$ be the endpoint space, which is a complex symplectic space with the symplectic form [:], see (A.26) through (A.32) above.

There exists a self-adjoint extension $T$ on $T_{0}$ (so $T$ is necessarily a restriction of $T_{1}$ ) if and only if there exists a complete Lagrangian subspace $\mathbf{L} \subset \mathbf{S}$, and this occurs if and only if the complex symplectic space $\mathbf{S}$ has excess Ex $=0$, i.e. $d^{+}=d^{-}=d$.

When $E x=0$, then $\operatorname{dim} \mathbf{S}=2 d \leq 2 n$ is even, and a Lagrangian subspace $\mathbf{L} \subset \mathbf{S}$ is complete if and only if $\operatorname{dim} \mathbf{L}=d$.

In this case, when $E x=0$, there exists a natural bi-unique correspondence between the set $\{T\}$ of all self-adjoint extensions $T$ of $T_{0}$ and the set $\{\mathbf{L}\}$ of all complete Lagrangian subspaces $\mathbf{L}$ of the endpoint space $\mathbf{S}$. Namely, for each such self-adjoint operator $T$ on domain $D(T) \subset L^{2}(\mathcal{J})$, which is an extension of $T_{0}$ on $D\left(T_{0}\right)$, the corresponding complete Lagrangian subspace $\mathbf{L}$ is defined by

$$
\mathbf{L}=D(T) / D\left(T_{0}\right),
$$


SO

$$
D(T)=c_{1} f_{1}+\cdots+c_{d} f_{d}+D\left(T_{0}\right) .
$$

Here $\left\{\mathbf{f}_{1}, \ldots, \mathbf{f}_{d}\right\}$ is any basis of $\mathbf{L}$, with any corresponding representative functions $f_{1}, \ldots, f_{d} \in D\left(T_{1}\right)$, and $c_{1}, \ldots, c_{d}$ are arbitrary complex numbers.

The special case $\mathbf{S}=0$, so $\mathbf{L}=0$ is the unique complete Lagrangian subspace, occurs if and only if $T_{0}=T_{1}$, so $T=T_{0}$ on $D(T)=D\left(T_{0}\right)$ is the unique self-adjoint extension of the self-adjoint operator $T_{0}$.

The GKN-Theorem follows immediately, as Corollary 1 of Theorem 1, from the developments in Section 2 above, once the preliminary facts concerning $T_{0} \subseteq T_{1}$ are established in the classical theory of linear ordinary differential equations [DS]. The case $n=1$ is treated specially in [EM1].

Generalizations of these results for quasi-differential expressions and for other complex Hilbert spaces $L^{2}(\mathcal{J} ; w)$ (defined with respect to other regular measures $w d x$ on the interval $\mathcal{J}$, see $[\mathrm{EV}],[\mathrm{EZ}])$ are developed in a monograph $[\mathrm{EM}]$ by the authors of this paper.

\section{REFERENCES}

[AG] Akhiezer, N.I. and Glazman, I.M., Theory of linear operators in Hilbert space: volumes I and II, Pitman and Scottish Academic Press, London, 1981; translated from the third Russian edition of 1977. MR 83i:47001

[AM] Abraham, R. and Marsden, J.E., Foundations of mechanics, 2nd ed., Benjamin/Cummings Publ. Co., Reading, Mass., 1978. MR 81e:58025

[DS] Dunford, N. and Schwartz, J.T., Linear operators: Part II, Wiley, New York, 1963. MR 32:6181

[EI] Everitt, W.N., On the deficiency index problem for ordinary differential operators 19101977, Proceedings of The 1977 Uppsala International Conference: Differential Equations, 62-81, Published by the University of Uppsala, Sweden, 1977, distributed by Almquist and Wiksell International Stockholm, Sweden, pp. 62-81. MR 57:16788

[EV] L Linear ordinary quasi-differential expressions, Lecture notes for The Fourth International Symposium on Differential Equations and Differential Geometry, Beijing, Peoples' Republic of China, 1-28. (Department of Mathematics, University of Peking, Peoples' Republic of China; 1986).

[EM] Everitt, W.N. and Markus, L., Boundary Value Problems and Symplectic Algebra for Ordinary Differential and Quasi-Differential Operators, Math. Surveys and Monographs, vol. 61, Amer. Math. Soc., Providence, RI, 1999. CMP 99:03

[EM1] _ The Glazman-Krein-Naimark theorem for ordinary differential operators, in New Results on Operator Theory and Its Applications: The I. M. Glazman Memorial Volume, Operator Theory: Advances and Applications, vol. 98, Birkhäuser, Basel, 1997, pp. 118130. MR 99c: 47070

[ER] Everitt, W.N. and Race, D., Some remarks on linear ordinary quasi-differential expressions, Proc. London Math. Soc. (3) 54 (1987), 300-320. MR 88b:34014

[EZ] Everitt, W.N. and Zettl, A., Differential operators generated by a countable number of quasi-differential expressions on the real line, Proc. London Math. Soc. (3) 64 (1992), 524-544. MR 93k:34182

[GZ] Glazman, I.M., On the theory of singular differential operators, Uspehi Math. Nauk 40 (1950), 102-135; English translation in Amer. Math. Soc. Translations (1) 4 (1962), 331372. MR 13:254d; MR 15:327a

[MA] Markus, L., Hamiltonian dynamics and symplectic manifolds, Lecture Notes, University of Minnesota, University of Minnesota Bookstores, 1973, 1-256.

[MH] Meyer, K. and Hall, G.R., Introduction to Hamiltonian dynamical systems and the n-body problem, Springer, New York, 1992. MR 93b:70002

[MS] McDuff, D. and Salamon, D., Introduction to symplectic topology, Oxford Univ. Press, 1995. MR 97b:58062 
[NA] Naimark, M.A., Linear differential operators: Part II, Ungar, New York, 1968; translated from the manuscript of the second Russian edition, "Nauka", Moscow, 1969. MR 41:7185

[TU] Turrittin, H.L., Convergent solutions of ordinary linear homogeneous differential equations in the neighborhood of an irregular singular point. Acta Math. 93, 27-66 (1955). MR 16:925a

Department of Mathematics and Statistics, University of Birmingham, Birmingham B15 2TT, England, United Kingdom

E-mail address: w.n.everitt@bham.ac.uk

Department of Mathematics, University of Minnesota, Minneapolis, Minnesota 55455

E-mail address: markus@math.umn.edu 